\title{
A VARIATIONAL MODEL FOR INFINITE PERIMETER SEGMENTATIONS BASED ON LIPSCHITZ LEVEL SET FUNCTIONS: DENOISING WHILE KEEPING FINELY OSCILLATORY BOUNDARIES *
}

\author{
M. BARCHIESI ${ }^{\dagger}$, S. H. KANG ${ }^{\ddagger}$, T. M. LE ${ }^{\S}$, M. MORINI ${ }^{\llbracket}$, AND M. PONSIGLIONE"
}

\begin{abstract}
We propose a new model for segmenting piecewise constant images with irregular object boundaries: a variant of the Chan-Vese model [10], where the length penalization of the boundaries is replaced by the area of their neighborhood of thickness $\varepsilon$. Our aim is to keep fine details and irregularities of the boundaries while denoising additive Gaussian noise. For the numerical computation we revisit the classical $B V$ level set formulation [24] considering suitable Lipschitz level set functions instead of $B V$ ones.
\end{abstract}

Key words. Denoising, Segmentation, Variational Methods, Level Set.

AMS subject classifications. 68U10, 35A15, 65C20.

1. Introduction. One of the most well known variational segmentation models is the one proposed by Mumford and Shah in [21], based on the minimization of the following functional:

$$
\mathcal{E}_{m s}\left[u, \Gamma \mid u_{0}\right]=\mathcal{H}^{1}(\Gamma)+\alpha \int_{\Omega \backslash \Gamma}|\nabla u|^{2} d x+\lambda \int_{\Omega}\left(u-u_{0}\right)^{2} d x .
$$

Here $\Omega$ is a bounded Lipschitz domain (e.g., a rectangle), $u_{0}: \Omega \rightarrow \mathbb{R}_{+} \cup\{0\}$ is the given image, $\Gamma \in \Omega$ denotes the edge set of the ideal image $u$, and $\mathcal{H}^{1}$ denotes the 1-dimensional Hausdorff measure, extending the classical notion of length. This functional is well defined on the pairs $(u, \Gamma)$ with $u \in W^{1,2}(\Omega \backslash \Gamma), \mathcal{H}^{1}(\Gamma)<\infty$, and $\Gamma$ being relatively closed in $\Omega$, provided that the given image $u_{0}$ belongs to $L^{2}(\Omega)$.

One other well-known work includes the Chan-Vese model [10], which successfully implemented the piecewise constant segmentation (i.e., a constrained version of the Mumford-Shah functional where only functions taking two values are taken into account), using the level-set method. In the context of two-phase segmentation the corresponding energy looks like

$$
\mathcal{E}_{c v}\left[v, \Gamma \mid u_{0}\right]=\mathcal{H}^{1}(\Gamma)+\lambda \sum_{i=1}^{2} \int_{U_{i}}\left|u_{0}-c_{i}\right|^{2},
$$

*This work was supported by the Hausdorff Research Institute for Mathematics (HIM) through the Junior Program on Analysis in 2008.

${ }^{\dagger}$ BCAM, Bizkaia Technology Park, Building 500, 48160 Derio, Spain (barchiesi@bcamath.org). The research of this author was also supported by the Center for Nonlinear Analysis (NSF Grants No. DMS-0405343 and DMS-0635983).

${ }^{\ddagger}$ School of Mathematics, 686 Cherry Street NW, Georgia Institute of Technology, Atlanta, GA 30332-0160, USA (kang@math.gatech.edu). The research of this author was also supported by NSF Grant No. DMS-0908517.

$\S$ Department of Mathematics, Yale University, 442 Dunham Lab., 10 Hillhouse Ave., New Haven, CT 06511, USA (triet.le@yale.edu). The research of this author was also supported by NSF Grant No. DMS-0809270 and by ONR N00014-09-1-0108.

๑SISSA, Via Bonomea 265, 34136 Trieste, Italy (morini@sissa.it).

"Dipartimento di Matematica "G. Castelnuovo", Università di Roma "La Sapienza", Piazzale A. Moro 2, 00185 Roma, Italy (ponsigli@mat.uniroma1.it). 
where $v: \Omega \rightarrow\{0,1\}$ is discontinuous on $\Gamma$ and divides $\Omega$ in two sets $U_{1}:=\{v=1\}$ and $U_{2}:=\{v=0\}$, and $c_{1}$ and $c_{2}$ are the averages of $u_{0}$ on $U_{1}$ and $U_{2}$, respectively. The function $u:=c_{1} \chi_{U_{1}}+c_{2} \chi_{U_{2}}$ represents the segmented image.

Following these variational segmentation models, numerous extensions and properties have been studied, such as $[4,7,11,16,18,19,20,25,26,27,28]$.

In this paper, as an attempt to segment objects with irregular boundaries, we propose and study a variant of the Mumford-Shah functional (1.1) (following the Chan-Vese approach (1.2) for piecewise constant segmentation), replacing the length term $\mathcal{H}^{1}(\Gamma)$ with the area of the set

$$
\varepsilon-\Gamma:=\bigcup_{x \in \Gamma} B_{\varepsilon}(x) .
$$

We call this set the $\varepsilon$-neighborhood of the edge set $\Gamma$. By introducing this new lengthscale parameter $\varepsilon$ in the model, we aim to capture rough boundaries of the main objects in the image $u_{0}$, while achieving at the same time the denoising effect. The heuristic behind this feature is that, on dilute noise, the area of the $\varepsilon$-neighborhood acts as an additive measure, while on fine oscillatory boundaries it is strictly subadditive. In view of this behavior, the $\varepsilon$-neighborhood term penalizes the presence of isolated connected components in the segmented picture, thus removing dilute noise, while preserving finely oscillating boundaries of the main objects in the picture, even allowing for infinite perimeter segmentations.

We analyze the properties of the proposed model focusing on two-phase segmentation. We propose numerical approximations of the model, based on the use of Lipschitz level set functions, and present various experiments.

Let $\mathcal{L}^{2}$ be the 2-dimensional Lebesgue measure. In order to approximate the $\varepsilon$-neighborhood, we first notice that the term $\mathcal{L}^{2}(\varepsilon-\Gamma)$ can be rewritten as

$$
\mathcal{L}^{2}(\varepsilon-\Gamma)=\int_{\Omega} f_{0}\left(\frac{\operatorname{dist}(x, \Gamma(v))}{\varepsilon}\right) d x,
$$

where $f_{0}:=\chi_{[0,1]}$. A natural way of approximating this penalization term is given by replacing the discontinuous function $\chi_{[0,1]}$ with a smoother function $f$. This leads to the following functional

$$
\mathcal{E}_{f}(v):=\int_{\Omega} f\left(\frac{\operatorname{dist}(x, \Gamma(v))}{\varepsilon}\right) d x+\lambda \sum_{i=1}^{2} \int_{U_{i}}\left|u_{0}-c_{i}\right|^{2},
$$

where $f$ is a positive decreasing function. Examples of $f$ include

$$
f(t)=e^{-t^{k}}, \text { or } f(t)=\frac{1}{1+t^{k}}, \text { for } k \geq 1 .
$$

Now, the idea of our Lipschitz level set approach consists in working with level set functions $\phi$ that are signed distance functions from their zero level set $\Gamma$, so that

$$
f\left(\frac{\operatorname{dist}(\cdot, \Gamma))}{\varepsilon}\right)=f\left(\frac{|\phi|}{\varepsilon}\right) .
$$

In order to force $\phi$ to look like a signed distance function, we add the penalization

$$
\frac{1}{p} \int_{\Omega}|\nabla \phi|^{p} d x
$$


This term, as $p \rightarrow \infty$, enforces $|\nabla \phi| \leq 1$ (this power-law approximation has been proposed also in [17] as a model for dielectric breakdown), and in combination with the fitting term and the perimeter penalization ensures that any minimizing $\phi$ stays as a signed distance function from $\Gamma$.

The paper is organized as follows. In Section 2 we introduce our variational model and study its main properties. In particular we provide the relaxed energy functional and prove the existence of a minimizer of the original problem. One of the difficulties in this step is that minimizing sequences have in general unbounded perimeters, and may converge to a function which is not a characteristic function. To overcome this lack of compactness, we prove that any minimizer of the relaxed functional can be suitably modified to produce a minimizing characteristic function. Note that the minimizing set needs not be of finite perimeter, and this is indeed in agreement with the main goal of keeping most of the fine details of the original image while performing denoising. In the last part of the section we collect some heuristic computations to highlight the main features of our model as a comparison to the Chan-Vese model.

In Section 3 we present some approximations useful to the numerical implementation of the model. In the first one, we present our revisited Lipschitz level set method. The main novelty with respect to the classical $B V$ level set formulation [24], is that our method involves Lipschitz continuous level set functions, with uniformly bounded Lipschitz constant. These Lipschitz level set functions turn out to be useful in implementing the $\varepsilon$-neighborhood of the level set (see (1.4)). The second one is a phase-field approximation, inspired by the classical Ambrosio-Tortorelli functional [1], the novelty being again in the use of uniformly Lipschitz phase-field functions. Finally, we consider a variant of our variational model, involving the infinite Laplace operator, already used in [11] to deal with regularizations effect of the signed distance function $\phi$. The corresponding level set functions are still Lipschitz, but with Lipschitz constant determined by a suitable variational problem. This variant is an attempt of automatically selecting the length-scale $\varepsilon$. For all these approximations we perform a rigorous $\Gamma$-convergence analysis (we refer the reader to the monographs [5] and [15] devoted to the notion of $\Gamma$-convergence).

In Section 4 we present some numerical results. In particular, we compare the results obtained using our Lipschitz level set method with those obtained using the Chan-Vese implementation, based on the classical $B V$ level set formulation [24].

2. The proposed model. In this section we present our model and we show the existence of an optimal segmentation of $\Omega$ corresponding to the given image $u_{0}$. Moreover, we explore various properties of the model.

2.1. The set-up of the model. Let $\Omega \subset \mathbb{R}^{2}$ be a bounded Lipschitz open set representing the image domain, and $u_{0} \in L^{2}\left(\Omega ; \mathbb{R}_{+} \cup\{0\}\right)$ be a given image. We propose the following functional for piecewise constant two-phase segmentation with irregular boundaries (using the intensity fitting term from the Chan-Vese model):

$$
\mathcal{E}\left[v \mid u_{0}\right]:=\mathcal{L}^{2}(\varepsilon-\Gamma(v))+\lambda\left[\int_{U}\left(u_{0}-c_{1}\right)^{2}+\int_{\Omega \backslash U}\left(u_{0}-c_{2}\right)^{2}\right],
$$

where $\varepsilon, \lambda>0$ are two parameters, $v=\chi_{U} \in L^{\infty}(\Omega ;\{0,1\})$ is a segmentation of $\Omega$, $\Gamma=\Gamma(v)$ is the edge set of $v$, and $c_{1}$ and $c_{2}$ are the averages of $u_{0}$ on $U$ and $\Omega \backslash U$, respectively. If $v=\chi_{U}$ minimizes the functional (2.1), then we call $\{U, \Omega \backslash U\}$ a minimal partition (with respect $\varepsilon, \lambda$ and $u_{0}$ ). The segmented image is represented by the function $u:=c_{1} v+c_{2}(1-v)$. 
REMARK 1. Note that the averages $c_{1}$ and $c_{2}$ minimize the expression $\int_{U}\left(u_{0}-\right.$ $\left.c_{1}\right)^{2}+\int_{\Omega \backslash U}\left(u_{0}-c_{2}\right)^{2}$ among all $c_{1}, c_{2} \in \mathbb{R}$.

In order to give a suitable definition of the edge set $\Gamma=\Gamma(v)$, we need to take into account that $v$ is defined up to a negligible set (with respect to $\mathcal{L}^{2}$ ). Therefore we cannot merely set $\Gamma:=\partial U \backslash \partial \Omega$. Indeed, the $\varepsilon$-neighborhood of $\Gamma$ as defined in (1.3) is sensitive to negligible modifications: for instance supposing $U$ open and removing from it a point, we introduce new boundary and $\varepsilon-\Gamma$ changes. Consequently, with such definition of $\Gamma$, the functional (2.1) is not well posed on the class of the segmentations in $L^{\infty}(\Omega)$. To avoid any ambiguity, we need to consider a precise representative within the Lebesgue equivalence class of the given function. To this end, we recall that $x \in \Omega$ is a Lebesgue point of a given locally integrable function $v$ if there exists $L_{v}(x) \in[0,1]$ such that

$$
\lim _{r \rightarrow 0} \frac{1}{r^{2}} \int_{B(x, r)}\left|v(y)-L_{v}(x)\right| d y=0 .
$$

In this case, $L_{v}(x)$ is called the Lebesgue value of $v$ at $x$. Moreover, given $v$, almost every $x \in \Omega$ is a Lebesgue point for $v$, and satisfies $v(x)=L_{v}(x)$. Therefore, it is well defined the Lebesgue representative $\tilde{v}$ of $v$, given by

$$
\tilde{v}(x):= \begin{cases}L_{v}(x) & \text { if } x \text { is a Lebesgue point for } v ; \\ 0 & \text { elsewere. }\end{cases}
$$

Definition 2.1. The edge set of $v=\chi_{U}$ is defined as

$$
\Gamma(v)=\partial\left\{\tilde{v}^{-1}(1)\right\} \backslash \partial \Omega,
$$

where $\tilde{v}$ is the Lebesgue representative of $v$. In this way, $\varepsilon-\Gamma(v)$ is independent on the choice of the representative of $v$.

For the readers familiar with geometric measure theory, we mention that $\partial\left\{\tilde{v}^{-1}(1)\right\}$ coincides with the relative closure of the so-called essential boundary of $U$. Our choice of the precise representative $\tilde{v}$ yields that $\partial\left\{\tilde{v}^{-1}(1)\right\} \subseteq \partial\left\{w^{-1}(1)\right\}$ for any other representative $w$ of $v$. In other words, our definition gives the minimal boundary corresponding to $U$. Note also that, when $U$ is Lipschitz, then $\partial\left\{\tilde{v}^{-1}(1)\right\}$ coincides with the usual topological boundary $\partial U$.

REMARK 2 (The Minkowski content). Let $\Gamma$ be a subset of $\Omega$. Then the quantity

$$
\lim _{\varepsilon \rightarrow 0} \frac{\mathcal{L}^{2}(\varepsilon-\Gamma)}{2 \varepsilon}
$$

if exists, is called the Minkowski content of $\Gamma$. It is well known that if $\Gamma$ is regular (e.g. a smooth curve) then the Minkowski content of $\Gamma$ exists and coincides with the usual 1-dimensional measure $\mathcal{H}^{1}(\Gamma)$. Hence, the first term in our model (2.1) represents (as $\varepsilon \rightarrow 0$, and rescaled by $2 \varepsilon$ ) a good approximation of the classical perimeter.

Therefore, defining

$$
\mathcal{E}_{\varepsilon}\left[v \mid u_{0}\right]=\frac{1}{2 \varepsilon} \mathcal{L}^{2}(\varepsilon-\Gamma(v))+\lambda\left[\int_{U}\left(u_{0}-c_{1}\right)^{2}+\int_{\Omega \backslash U}\left(u_{0}-c_{2}\right)^{2}\right] d x,
$$

we expect that for small $\varepsilon$ the functionals $\mathcal{E}_{\varepsilon}$ behave like the Chan-Vese functional $\mathcal{E}_{c v}$ defined in (1.2). Indeed, by the analysis done in [6] it is possible to prove that the 
functionals $\mathcal{E}_{\varepsilon} \Gamma$-converge to $\mathcal{E}_{c v}$ as $\varepsilon \rightarrow 0$ (we refer also to [22] and to [14] for a similar analysis in the framework of fracture mechanics).

On the other hand, for fixed $\varepsilon, \mathcal{L}^{2}(\varepsilon-\Gamma)$ turns out to be much smaller than $2 \varepsilon \mathcal{H}^{1}(\Gamma)$ whenever $\Gamma$ presents fine oscillations at scales smaller than $\varepsilon$ (see Example 1). This relaxation in the perimeter penalization for small oscillations represents the main difference of our model with respect to the $C V$-model.

EXAMPLE 1. Let $\Omega=(0,1)^{2}$ and let $u_{0}$ be defined for all $(x, y) \in \Omega$ as

$$
u_{0}(x, y)=\left\{\begin{array}{cc}
1 & y<1+\frac{x}{2} \sin \left(\frac{1}{x}\right) \\
0 & \text { otherwise }
\end{array}\right.
$$

In this case, $\Gamma=\left\{(x, y): y=1+\frac{x}{2} \sin \left(\frac{1}{x}\right)\right\}$. A simple calculation shows that $\mathcal{H}^{1}(\Gamma)=$ $\infty$. On the other hand, $\mathcal{L}(\varepsilon-\Gamma) \leq 1$ for all $\varepsilon>0$.

Another motivation to consider $\mathcal{L}^{2}(\varepsilon-\Gamma)$ is the case when $\Gamma$ corresponds to fractal boundaries. For instance, consider a Koch snowflake (we refer the reader to [13] for its definition). Its boundary $\Gamma$ clearly satisfies $\mathcal{H}^{1}(\Gamma)=\infty$, while for any $\varepsilon>0, \mathcal{L}^{2}(\varepsilon-\Gamma)$ is finite. Finally, we notice that also the Minkowski content of $\Gamma$ is infinite, and indeed the asymptotic behavior of $\mathcal{L}^{2}(\varepsilon-\Gamma)$ as $\varepsilon \rightarrow 0$ is related to the fractal dimension (the so called Minkowski dimension) of $\Gamma$. It would be interesting to understand if there is a natural way of tuning the parameters $\lambda$ and $\varepsilon$ in our model, related to the fractal dimension of $\Gamma$. However, this is beyond the scope of the present paper.

2.2. Existence of a minimizer. In this part we prove that the energy $\mathcal{E}$ in (2.1) admits a minimal partition $\{U, \Omega \backslash U\}$ of $\Omega$. Our approach follows the lines of the direct method of the calculus of variations. However, since the model allows for oscillatory boundaries, the minimizing sequence may not have finite perimeter, and hence we can not apply standard compactness arguments for sets with finite perimeter. The minimal partition itself could have infinite perimeter, and this is indeed the main feature of our model. In the following, to simplify the notation we neglect the dependence on $u_{0}$.

The main ingredient in the proof of the existence of a minimizer is given by providing a relaxation formula for the functional $\mathcal{E}$. For this purpose, we define the relaxation $\overline{\mathcal{E}}: L^{\infty}(\Omega ;[0,1]) \rightarrow \mathbb{R}$ of $\mathcal{E}$ as

$$
\overline{\mathcal{E}}(v):=\inf \left\{\liminf _{n} \mathcal{E}\left(v_{n}\right), v_{n} \in L^{\infty}(\Omega ;\{0,1\}), v_{n} \stackrel{*}{\rightarrow} v \text { in } L^{\infty}(\Omega ;[0,1])\right\} .
$$

Since $L^{\infty}(\Omega ;[0,1])$ is compact with respect to the weak* topology, it is a standard fact (we refer the reader to [8]) that the relaxation $\overline{\mathcal{E}}$ of $\mathcal{E}$ admits a minimizer, and that

$$
\inf _{v \in L^{\infty}(\Omega ;\{0,1\})} \mathcal{E}(v)=\min _{v \in L^{\infty}(\Omega ;[0,1])} \overline{\mathcal{E}}(v)
$$

In order to give an explicit formula for the functional $\overline{\mathcal{E}}$, we need to generalize the definition of $\Gamma(v)$ introduced in $(2.2)$ to functions $v \in L^{\infty}(\Omega ;[0,1])$ :

$$
\Gamma(v):=\left(\partial\left\{\tilde{v}^{-1}(0)\right\} \cup \partial\left\{\tilde{v}^{-1}(1)\right\} \cup \tilde{v}^{-1}((0,1))\right) \backslash \partial \Omega,
$$

where we recall that $\tilde{v}$ is the Lebesgue representative of $v$. Denoting by $A^{0}$ the interior of $\tilde{v}^{-1}(0)$ and by $A^{1}$ the interior of $\tilde{v}^{-1}(1)$, we have $\Gamma(v)=\Omega \backslash\left(A^{0} \cup A^{1}\right)$. In particular, the set $\Gamma(v)$ turns out to be relatively closed in $\Omega$. Observe that if $v \in L^{\infty}(\Omega ;\{0,1\})$, then the notions of $\Gamma(v)$ defined in (2.2) and (2.5) agree. We finally mention that 
again this definition provides the minimal edge set within the Lebesgue equivalence class of $v$.

In the following, we will need a stability result for the penalization term $\mathcal{L}^{2}(\varepsilon-K)$ with respect to the Hausdorff convergence. To this end, we recall that a sequence of closed sets $K_{n}$ converges to a closed set $K$ in the sense of Hausdorff convergence if the following two properties hold:

i) for every positive $\delta>0$, and for $n$ large enough (depending on $\delta$ ), we have $K_{n} \subseteq \delta-K$

ii) for every positive $\delta>0$, and for $n$ large enough (depending on $\delta$ ), we have $K \subseteq \delta-K_{n}$.

It is well known (see [13]) that any sequence of equibounded closed sets $K_{n}$ admits a convergent subsequence. The following stability result is a direct consequence of the definition of Hausdorff convergence.

LEMMA 2.2. Let $K_{n}$ be a sequence of closed sets converging to a closed set $K$ in the sense of Hausdorff convergence. Then $\mathcal{L}^{2}\left(\varepsilon-K_{n}\right) \rightarrow \mathcal{L}^{2}(\varepsilon-K)$ as $n \rightarrow \infty$.

We are in a position to give an explicit formula for the relaxation $\overline{\mathcal{E}}$ of $\mathcal{E}$.

Proposition 2.3. For all $v \in L^{\infty}(\Omega ;[0,1])$ the following relaxation formula holds

$$
\overline{\mathcal{E}}(v)=\mathcal{L}^{2}(\varepsilon-\Gamma(v))+\lambda \int_{\Omega}\left[v\left(u_{0}-c_{1}(v)\right)^{2}+(1-v)\left(u_{0}-c_{2}(v)\right)^{2}\right] d x,
$$

with $c_{1}(v)$ and $c_{2}(v)$ defined by

$$
c_{1}(v):=\frac{\int_{\Omega} v u_{0} d x}{\int_{\Omega} v d x} \quad \text { and } \quad c_{2}(v):=\frac{\int_{\Omega}(1-v) u_{0} d x}{\int_{\Omega}(1-v) d x}
$$

REMARK 3. Note that the values $c_{1}(v)$ and $c_{2}(v)$ are obtained by minimizing the expression $\int_{\Omega}\left[v\left(u_{0}-c_{1}\right)^{2}+(1-v)\left(u_{0}-c_{2}\right)^{2}\right]$ in $c_{1}, c_{2} \in \mathbb{R}$.

Proof. We will divide the proof into two steps, where we will prove that (2.6) holds with the equality replaced by the inequalities " $\leq$ " and " $\geq$ " respectively.

Step $1(\leq)$. Let $A^{0}$ be the interior of $\tilde{v}^{-1}(0), A^{1}$ the interior of $\tilde{v}^{-1}(1)$, so that $\Gamma(v)=\Omega \backslash\left(A^{0} \cup A^{1}\right)$. By standard arguments, it is possible to construct a sequence $w_{n} \in L^{\infty}(\Gamma(v) ;\{0,1\})$ such that $\left.w_{n} \stackrel{*}{\rightarrow} v\right|_{\Gamma(v)}$ in $L^{\infty}(\Gamma(v) ;[0,1])$. Define

$$
v_{n}(x):= \begin{cases}0 & \text { if } x \in A^{0} \\ 1 & \text { if } x \in A^{1} \\ w_{n} & \text { if } x \in \Gamma(v)\end{cases}
$$

Clearly, we have $v_{n} \stackrel{*}{\rightarrow} v$ in $L^{\infty}(\Omega ;[0,1])$, and hence by definition $\overline{\mathcal{E}}(v) \leq \liminf \mathcal{E}\left(v_{n}\right)$. Notice that by the very definition of $v_{n}$, we have $\Gamma\left(v_{n}\right) \subseteq \Gamma(v)$, and in particular

$$
\mathcal{L}^{2}\left(\varepsilon-\Gamma\left(v_{n}\right)\right) \leq \mathcal{L}^{2}(\varepsilon-\Gamma(v))
$$


Let $U\left(v_{n}\right):=v_{n}^{-1}(1)$. In view of (2.9) and of Remark (1), we have

$$
\begin{aligned}
\overline{\mathcal{E}}(v) & \leq \liminf _{\mathcal{E}}\left(v_{n}\right) \\
& \leq \liminf _{n}\left(\mathcal{L}^{2}\left(\varepsilon-\left(\Gamma\left(v_{n}\right)\right)+\lambda\left[\int_{U\left(u_{n}\right)}\left(u_{0}-c_{1}(v)\right)^{2} d x+\int_{\Omega \backslash U\left(u_{n}\right)}\left(u_{0}-c_{2}(v)\right)^{2} d x\right]\right)\right. \\
& \leq \lim _{n}\left(\mathcal{L}^{2}\left(\varepsilon-(\Gamma(v))+\lambda \int_{\Omega}\left[v_{n}\left(u_{0}-c_{1}(v)\right)^{2}+\left(1-v_{n}\right)\left(u_{0}-c_{2}(v)\right)^{2}\right] d x\right)\right. \\
& =\mathcal{L}^{2}(\varepsilon-\Gamma(v))+\lambda \int_{\Omega}\left[v\left(u_{0}-c_{1}(v)\right)^{2}+(1-v)\left(u_{0}-c_{2}(v)\right)^{2}\right] d x .
\end{aligned}
$$

Step $2(\geq)$. Let $v_{n}$ be a sequence in $L^{\infty}(\Omega ;\{0,1\})$ such that $v_{n} \stackrel{*}{\rightarrow} v$ and $\overline{\mathcal{E}}(v)=$ $\lim \mathcal{E}\left(v_{n}\right)$. Up to a subsequence (that we will not relabel) we can assume that $\overline{\Gamma\left(v_{n}\right)} \rightarrow$ $\Gamma$ in the sense of Hausdorff for some closed set $\Gamma$. First let us show that $\Gamma(v) \subseteq \Gamma$. Indeed, let $x \in \Omega \backslash \Gamma$. Therefore, by the definition of Hausdorff convergence there exists $\delta>0$ such that $\Gamma\left(v_{n}\right) \cap B_{\delta}(x)=\emptyset$ for $n$ large enough. This implies that either $v_{n} \equiv 0$, or $v_{n} \equiv 1$ on $B_{\delta}(x)$, and these properties are clearly inherited by $v$. In conclusion, we have that $x$ is in the interior of the set $\left\{v^{-1}(0)\right\} \cup\left\{v^{-1}(1)\right\}$, and hence $x$ does not belong to $\Gamma(v)$. By Lemma 2.2 we deduce that

$$
\lim _{n} \mathcal{L}^{2}\left(\varepsilon-\Gamma\left(v_{n}\right)\right)=\lim _{n} \mathcal{L}^{2}\left(\varepsilon-\overline{\Gamma\left(v_{n}\right)}\right)=\mathcal{L}^{2}(\varepsilon-\Gamma) \geq \mathcal{L}^{2}(\varepsilon-\Gamma(v)) .
$$

By the continuity with respect to the weak* topology of the coefficients $c_{1}(\cdot)$ and $c_{2}(\cdot)$, and in view also of $(2.10)$, we get

$$
\begin{aligned}
\overline{\mathcal{E}}(v) & =\lim \mathcal{L}^{2}\left(\varepsilon-\left(\Gamma\left(v_{n}\right)\right)+\lambda \int_{\Omega}\left[v_{n}\left(u_{0}-c_{1}\left(v_{n}\right)\right)^{2}+\left(1-v_{n}\right)\left(u_{0}-c_{2}\left(v_{n}\right)\right)^{2}\right] d x\right. \\
& =\mathcal{L}^{2}(\varepsilon-(\Gamma))+\lambda \int_{\Omega}\left[v\left(u_{0}-c_{1}(v)\right)^{2}+(1-v)\left(u_{0}-c_{2}(v)\right)^{2}\right] d x \\
& \geq \mathcal{L}^{2}(\varepsilon-(\Gamma(v)))+\lambda \int_{\Omega}\left[v\left(u_{0}-c_{1}(v)\right)^{2}+(1-v)\left(u_{0}-c_{2}(v)\right)^{2}\right] d x .
\end{aligned}
$$

This concludes the proof of the proposition.

We are in a position to give the main result of the Section.

THEOREM 2.4. For any given original image $u_{0}$ in $L^{2}\left(\Omega ; \mathbb{R}_{+} \cup 0\right)$, the functional $\mathcal{E}$ defined in (2.1) admits a minimizer.

Proof. Let $\bar{v}$ be a minimizer for $\overline{\mathcal{E}}$. Setting

$$
v(x):= \begin{cases}\bar{v}(x) & \text { on } \bar{v}^{-1}(\{0\} \cup\{1\}), \\ 0 & \text { on } x \in \Omega:\left|u_{0}(x)-c_{1}(\bar{v})\right| \leq\left|u_{0}(x)-c_{2}(\bar{v})\right|, \\ 1 & \text { on } x \in \Omega:\left|u_{0}(x)-c_{1}(\bar{v})\right|>\left|u_{0}(x)-c_{2}(\bar{v})\right|,\end{cases}
$$

it is easy to see that $v$ is also a minimizer of $\overline{\mathcal{E}}$ (since, passing from $\bar{v}$ to $v$, both addends in (2.6) do not increase). Finally, in view of (2.4) we conclude that $v$ minimizes $\mathcal{E}$. $\mathbf{\square}$

REMARK 4 (The instability of segmentation in $\Gamma$ ). A theoretical drawback of our model is that a minimizer $\bar{v}$ of $\overline{\mathcal{E}}$ could in general exhibit a diffuse region $I(\bar{v}):=\{x \in$ $\Omega: 0<\bar{v}<1\} \subseteq \Gamma(\bar{v})$. In the proof of Theorem 2.4 we have seen that we can always modify $\bar{v}$ on $I(\bar{v})$ into a characteristic function keeping its minimality. On the other hand, we expect in $I(\bar{v})$ to have instability of the minimization process, since in this region the minimizer is not unique. Therefore, two natural questions arise: 
1) How large could the instability region be?

2) Are there natural assumptions on the original image $u_{0}$ ensuring that $I(\bar{v})=$ $\emptyset$, i.e., that any minimizer of $\overline{\mathcal{E}}$ takes two values?

Concerning the first question, we observe that by minimality we always deduce the following bound for $\Gamma(\bar{v})$ :

$$
\mathcal{L}^{2}(\Gamma(v)) \leq \mathcal{L}^{2}(\varepsilon-(\Gamma(v))) \leq \mathcal{E}(v) \leq \mathcal{E}\left(\chi_{\Omega}\right) \leq \lambda\left\|u_{0}\right\|_{2}^{2},
$$

that clearly gives a bound for $I(v) \subseteq \Gamma(v)$. Passing to the second question, we notice that by minimality we necessarily have

$I(v) \subseteq\left\{x \in \Omega:\left|u_{0}(x)-c_{2}(v)\right|=\left|u_{0}(x)-c_{1}(v)\right|\right\}=\left\{x \in \Omega: u_{0}(x)=\frac{c_{2}(v)+c_{1}(v)}{2}\right\}$.

Therefore, if for instance $u_{0}$ does not have flat parts, i.e., $u_{0}^{-1}(t)$ has measure zero for every $t \in \mathbb{R}$, then we deduce that $I(v)=\emptyset$. Another relevant assumption ensuring $I(v)=\emptyset$ is that $u_{0}$ takes only two values (because in this case none of these values can coincide with $\left.\left(c_{2}(v)+c_{1}(v)\right) / 2\right)$.

More in general, we expect that the presence of the instability region $I(v)$ is indeed a very rare event, and it should not be an obstacle for practical purposes.

REMARK 5 (A smooth variant of the proposed model). Here we discuss a possible variant of the proposed model. Let us observe that the relaxation $\overline{\mathcal{E}}$ of $\mathcal{E}$ can be rewritten as

$$
\mathcal{E}_{f_{0}}(v):=\int_{\Omega} f_{0}\left(\frac{\operatorname{dist}(x, \Gamma(v))}{\varepsilon}\right) d x+\lambda \int_{\Omega}\left[v\left(u_{0}-c_{1}(v)\right)^{2}+(1-v)\left(u_{0}-c_{2}(v)\right)^{2}\right] d x,
$$

where $f_{0}:=\chi_{[0,1]}$. A natural variant of our model, which could be convenient for numerical purposes, consists in replacing the discontinuous function $\chi_{[0,1]}$ with a smoother function $f$. This leads to the following functional in $L^{\infty}(\Omega ;[0,1])$ :

$$
\mathcal{E}_{f}(v):=\int_{\Omega} f\left(\frac{\operatorname{dist}(x, \Gamma(v))}{\varepsilon}\right) d x+\lambda \int_{\Omega}\left[v\left(u_{0}-c_{1}(v)\right)^{2}+(1-v)\left(u_{0}-c_{2}(v)\right)^{2}\right] d x
$$

where $c_{1}(v), c_{2}(v)$ are defined in (2.7) and $f$ is a positive non-increasing lower semicontinuous function. Examples of $f$ include

$$
f(t)=e^{-t^{k}}, \text { or } f(t)=\frac{1}{1+t^{k}}, \text { for } k \geq 1
$$

For large $k, f$ behaves more like $f_{0}$. The proof of the existence in $L^{\infty}(\Omega ;\{0,1\})$ of a minimizer for $\mathcal{E}_{f}$ goes along the lines of the proof given for the functional $\mathcal{E}_{f_{0}}$.

REMARK 6 (Invisible points for $\varepsilon-\Gamma(v)$ ). Let $v \in L^{\infty}(\Omega ;[0,1])$ and set

$$
N(v):=\left\{x \in \Omega: B_{\varepsilon}(x) \subseteq \varepsilon-\Gamma(v)\right\} .
$$

Then by construction we have $\mathcal{L}^{2}\left(\varepsilon-(\Gamma(v) \cup N(v))=\mathcal{L}^{2}(\varepsilon-(\Gamma(v))\right.$. Therefore, one can modify the function $v$ on $N(v)$ increasing the set $\Gamma$ but without changing the corresponding $\varepsilon$-neighborhood. The presence of such region could be a source of noise, since formation of boundaries are not penalized there. Moreover, the presence of $N(v)$, together with the set $I(v)$ discussed in Remark 4, may be a source of instability in our model.

Since the presence of $N(v)$ is made possible by the fact that the graph of the function $f_{0}$ in (2.11) has flat zones, a way to avoid the set $N(v)$ is then to consider the variant $\mathcal{E}_{f}$ in $(2.12)$, with $f$ strictly decreasing. 
2.3. Features and properties of our model. In this section we compare the properties of our model with the ones by Chan-Vese, focusing, in particular, on the following features.

- The denoising effect: for simplicity we will consider additive noise given by the presence in $u_{0}$ of many small isolated components, and we will compare the capability of the two models to remove them.

- The cornering effect: it is well known that the Chan-Vese model does not keep corners and tends to give them a round shape; we will compare the analogous effect with our proposed model.

- Resolution: namely the capability to distinguish between two close objects, instead of considering them as a single one.

- Oscillatory boundary: we will compare the capability of the two models of keeping finely oscillatory parts of the boundaries.

In Chan-Vese model, decreasing the parameter $\lambda$ allows to increase the denoising effect, although to the detriment of cornering, resolution and capability of keeping fine oscillations (and vice versa). The possibility of tuning on two parameters, $\varepsilon$ and $\lambda$, could result in a more performing model. In the following examples we try to clarify this point with some heuristic computations. More precisely we will link the parameter $\lambda_{c v}$ in the Chan-Vese model and the parameters $\lambda$ and $\varepsilon$ in our model to make the denoising effect of the same magnitude. Then, with this choice of the parameters, we will compare the cornering effect, the resolution and the capability of keeping oscillations.

We illustrate the situation with four examples. In the following $\Omega$ is a rectangle and $u_{0}=\chi_{U_{0}}$ for a certain $U_{0} \subset \Omega$. We will compare the energy functionals (2.1) and (1.2) on simple segmentations corresponding to some $v=\chi_{U}$, with $U \subset \Omega$ Lipschitz. Moreover, considering a slight simplification of the model, we will not optimize the constant $c_{1}$ and $c_{2}$, fixing instead $c_{1}=1, c_{2}=0$. In this case, the energy (1.2) reduces to the functional

$$
\mathcal{E}_{c v}\left[U \mid U_{0}\right]:=\mathcal{H}^{1}(\partial U \backslash \partial \Omega)+\lambda_{c v} \mathcal{L}^{2}\left(U \triangle U_{0}\right)
$$

while the energy in our model (2.1) reduces to

$$
\mathcal{E}\left[U \mid U_{0}\right]:=\mathcal{L}^{2}(\varepsilon-(\partial U \backslash \partial \Omega))+\lambda \mathcal{L}^{2}\left(U \triangle U_{0}\right) .
$$

Critical size for noise removal. Here we heuristically compute the critical size of the noise removed by the two models. Then we will link the corresponding parameters by enforcing the same critical size. To simplify matter we will assume that the noise is given by the union of many small balls, and we are looking for the critical radius $\delta$ (depending on the parameters) such that any ball of radius smaller than $\delta$ will be removed. Since we are interested in keeping fine details of the boundary of the given image, we assume the fidelity parameters $\lambda_{c v}$ and $\lambda$ to be very large. Finally, as a further simplification, we will assume that the mutual distance of these balls is larger than $2 \varepsilon$, so that their $\varepsilon$-neighborhoods are not overlapping; this allows us to reduce to the case of a single ball (see Fig. 2.1).

Consider $U_{0}=B_{\delta}(x) \subset \Omega$. In this situation the model (2.13) removes the ball $B_{\delta}(x)$ if and only if $\delta<2 / \lambda_{c v}$. Indeed, in terms of perimeter a ball of size $\delta$ weighs $2 \pi \delta$, while in terms of area it weighs $\lambda_{c v} \pi \delta^{2}$.

In our proposed model, assuming $\delta \leq \varepsilon$, we have $\mathcal{L}^{2}\left(\varepsilon-\partial B_{\delta}(x)\right)=\pi(\varepsilon+\delta)^{2}$. Therefore, after some elementary computations it turns out that, for the proposed 


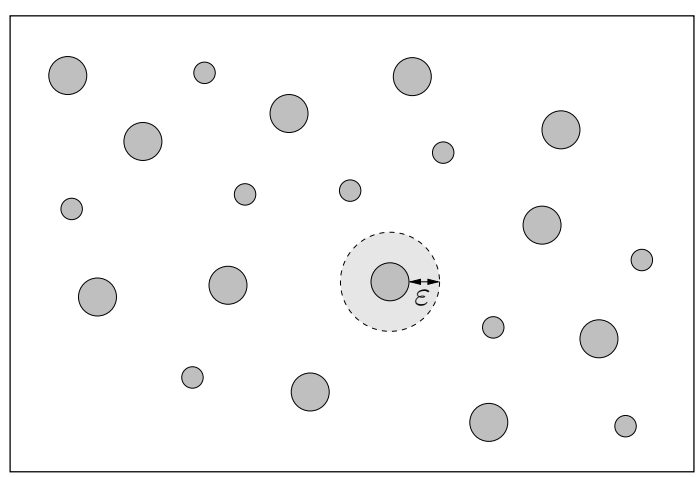

Figure 2.1. Denoising.

model (2.14), the critical size $\delta$ satisfies $\delta \approx c \varepsilon / \sqrt{\lambda}$, with $1<c \leq 2$ (the extremal cases corresponding to $\delta \rightarrow 0$ and to $\delta=\varepsilon$, respectively).

Now, enforcing the same denoising effect for the two models, i.e, the same critical size $\delta$, we deduce the relation between the parameters

$$
\frac{1}{\lambda_{c v}} \approx c \frac{\varepsilon}{\sqrt{\lambda}}
$$

Cornering. Here we compare the smoothing effect of the two models, assuming they perform the same denoising, i.e., that $(2.15)$ holds. For this purpose, we take $U_{0}=Q$, with $Q$ the unitary cube, and we consider the one-parameter family of competitors $U_{\delta}$ (with $\delta>0$ ) obtained by $U_{0}$ replacing the corners with circular arcs of radius $\delta$ (see Fig. 2.2).

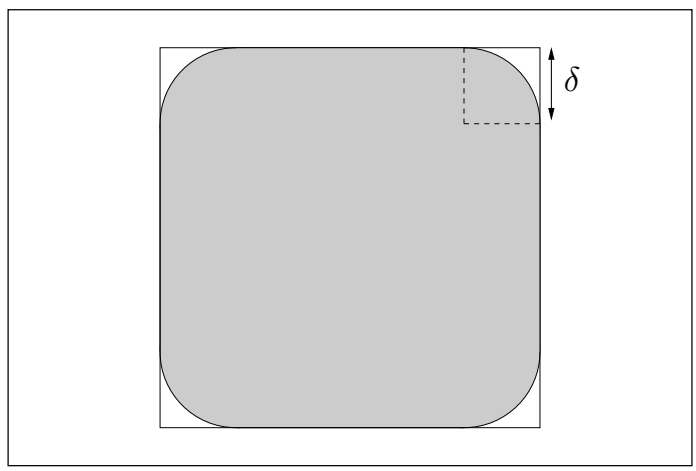

Figure 2.2. Cornering.

Then minimizing the corresponding energy functionals with respect to $\delta$ gives the magnitude of the smoothing effect for the two models.

An elementary computation leads to the following expression:

$$
\mathcal{E}_{c v}\left[U_{\delta} \mid U_{0}\right]=4-2(4-\pi) \delta+\lambda_{c v}(\pi-4) \delta^{2} .
$$

Minimizing with respect to $\delta$, we obtain the smoothing radius $\delta_{c v}=1 / \lambda_{c v}$.

Passing to our proposed model, we have

$$
\mathcal{E}\left[U_{\delta} \mid U_{0}\right]=(1+2 \varepsilon)^{2}+(1-2 \varepsilon)^{2}-(4-\pi)(\delta+\varepsilon)^{2}+\lambda(4-\pi) \delta^{2} .
$$


Minimizing in $\delta$, we obtain the smoothing radius for our proposed model $\delta=\varepsilon /(\lambda-1)$. Finally, enforcing (2.15) we deduce for $\lambda$ large

$$
\delta=\frac{\varepsilon}{\lambda-1} \approx \frac{\varepsilon}{\lambda}=\frac{1}{2 \lambda_{c v}} \frac{1}{\sqrt{\lambda}}=\delta_{c v} \frac{1}{2 \sqrt{\lambda}} .
$$

Summarizing, the above heuristic computation shows that our model seems to better preserve corners while performing the same denoising.

Resolution. Let $U_{0}$ be a strip of unitary height and length $l$ with a crevice of size $\delta \ll 1$ (see Fig. 2.3). Moreover, let $U$ be the rectangle obtained by filling the crevice of $U_{0}$.

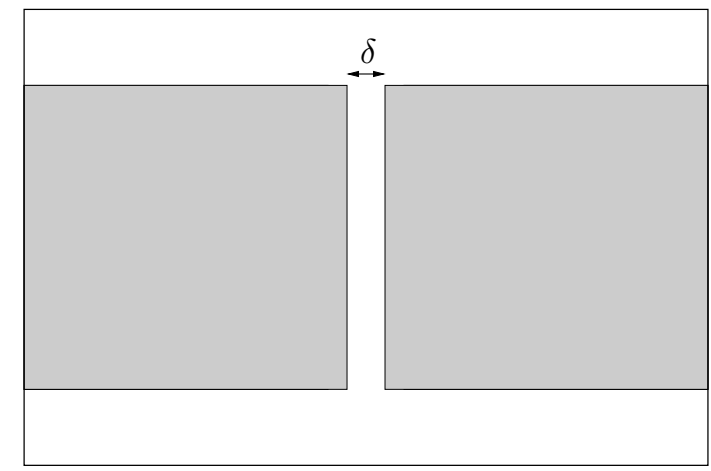

FiguRe 2.3. Resolution.

We will compare now the resolution of the two models, understood as the capability of keeping the crevice without filling it. To this end, we compare the energy of $U$ and $U_{0}$ for the two models, obtaining in this way the critical size $\delta$ of the crevice. Concerning the Chan-Vese model we have

$$
\mathcal{E}_{c v}\left[U \mid U_{0}\right]=2 l+\lambda_{c v} \delta, \quad \mathcal{E}_{c v}\left[U_{0} \mid U_{0}\right]=2(l-\delta)+2 .
$$

Therefore, the critical $\delta$ is given by

$$
\delta_{c v}=\frac{2}{\lambda_{c v}+2} \approx \frac{2}{\lambda_{c v}} .
$$

Passing to our model, assuming $\delta<2 \varepsilon$ a straightforward computation gives

$$
\mathcal{E}\left[U \mid U_{0}\right]=4 \varepsilon l+2 \pi \varepsilon^{2}+\lambda \delta, \quad \mathcal{E}\left[U_{0} \mid U_{0}\right]=4 \varepsilon l+2 \pi \varepsilon^{2}+\delta+2 \varepsilon+O(\varepsilon \delta),
$$

where $O(\varepsilon \delta)$ is such that $O(\varepsilon \delta) /(\varepsilon \delta) \leq C$. By comparison, the critical $\delta$ in our model is given by $\delta \approx 2 \varepsilon /(\lambda-1) \approx 2 \varepsilon / \lambda$. Finally, enforcing (2.15), we get

$$
\delta \approx \frac{2 \varepsilon}{\lambda} \approx \frac{2}{\lambda_{c v} \sqrt{\lambda}} \approx \frac{\delta_{c v}}{\sqrt{\lambda}} .
$$

This computation shows that on equal denoising, our model should guarantee a better resolution. 


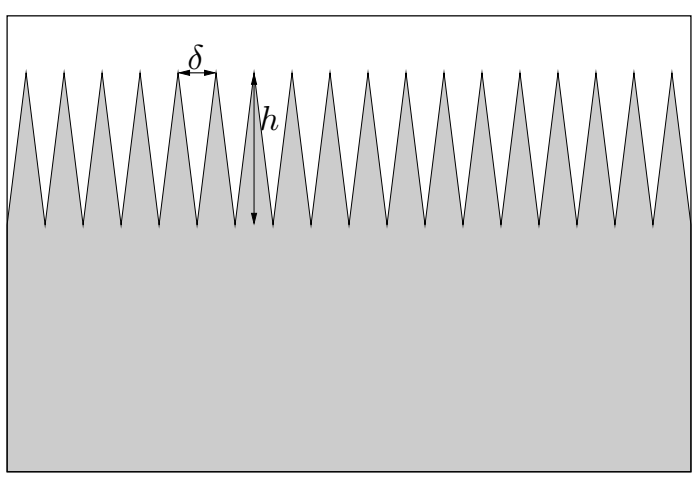

(a)

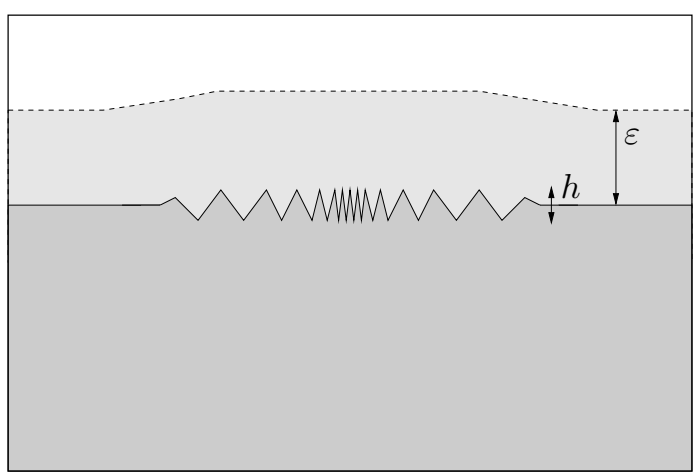

(b)

Figure 2.4. Oscillatory boundary.

Fine oscillatory boundaries. Finally, we compare the capability of preserving fine oscillatory boundaries for the two models. Let $U$ be a strip of unitary height and length $l$. Moreover, let $U_{0}$ be the previous strip with in addition, along one of the sides of length $l$, a saw-toothed set constituted by triangles of base $\delta$ and height $h$ as in Fig. 2.4(a).

We assume $\delta$ small compared to $l$. Since the number of the triangles of the saw-toothed region is approximately $l / \delta$, we have

$$
\mathcal{E}_{c v}\left[U \mid U_{0}\right] \approx l+\frac{\lambda_{c v} h l}{2}, \quad \mathcal{E}_{c v}\left[U_{0} \mid U_{0}\right] \approx \frac{2 l}{\delta} \sqrt{h^{2}+\left(\frac{\delta}{2}\right)^{2}},
$$

from which we easily deduce that the critical size of the frequency of the oscillations is given by

$$
\delta_{c v} \approx \frac{4 h}{\sqrt{3+4 \lambda_{c v} h+\lambda_{c v}^{2} h^{2}}} .
$$

This suggests that, in the case of the fidelity parameter $\lambda_{c v}$ quite larger than the inverse of the amplitude $h$ of the oscillation $\left(\lambda_{c v} h \gg 1\right)$, the sets close to $U_{0}$ are energetically favorable in the Chan-Vese model when the frequency $\delta$ of oscillation is larger than $4 / \lambda_{c v}$. For $\delta<4 / \lambda_{c v}$ we have instead that the set $U$ is energetically favorable, 


\begin{tabular}{l|ll} 
Model & Chan-Vese & Proposed \\
Denoising & $\delta_{c v}=\frac{2}{\lambda_{c v}}$ & $\delta=\delta_{c v}$ \\
Cornering & $\delta_{c v}=\frac{1}{\lambda_{c v}}$ & $\delta=\frac{1}{2 \sqrt{\lambda}} \delta_{c v}$ \\
Resolution & $\delta_{c v}=\frac{2}{\lambda_{c v}}$ & $\delta=\frac{1}{\sqrt{\lambda}} \delta_{c v}$ \\
Oscillatory boundaries & $\delta_{c v}=\frac{4}{\lambda_{c v}}$ & every $\delta<<\varepsilon$
\end{tabular}

$$
\begin{gathered}
\text { TABLE } 2.1 \\
\text { Comparison with } \frac{1}{\lambda_{c v}} \approx \frac{\varepsilon}{\sqrt{\lambda}} .
\end{gathered}
$$

and indeed we expect that the minimal segmentation cuts-off the vertical oscillations, decreasing the scale $h$ and smoothing the saw-toothed set. In the case $\lambda h \ll 1$, the sets close to $U_{0}$ are energetically favorable when the frequency of oscillation is at least larger than $4 h / \sqrt{3}$, that is the same order of the amplitude $h$.

Passing to our model the picture changes drastically, since we have

$$
\mathcal{E}\left[U \mid U_{0}\right] \approx 2 \varepsilon l+\frac{\lambda h l}{2}, \quad \mathcal{E}\left[U_{0} \mid U_{0}\right] \approx(2 \varepsilon+h) l .
$$

Therefore, for any given $\lambda \geq 2$ we could expect that the minimum of our model is closer to $U_{0}$ than to $U$. Moreover, when the oscillatory parts of the boundary are at a scale smaller than $\varepsilon$, we aspect that the saw-toothed set is substantially kept because each perturbation flattening the boundary does not modify substantially the $\varepsilon$-neighborhood. Similarly, the closeness of the minimum to $U_{0}$ should not be dependent by the frequency $\delta$ of the oscillation, because the $\varepsilon$-neighborhood remains almost the same (see Fig. 2.4(b)). This fact is indeed one of the main features of our model.

REMARK 7 (Features of our model). In Table 1 we have summarized the features of our model. In particular, assuming that the parameters in our model and in the Chan-Vese model are chosen to perform similar denoising, then our model turns out to perform better concerning the capability of keeping fine details of the boundary. We stress anyway that such heuristic computations have been performed on ideal images, and through an asymptotic analysis assuming $\lambda$ very big and the noise very dilute.

On the other hand, in our simulations we will see that for real images we can fix the parameters of our model in order to perform at the same time stronger denoising than Chan-Vese model, keeping finer details of the boundaries.

REMARK 8 (Dense noise). The link between the parameters (2.15) has been obtained by heuristic computations assuming that the noise is made of balls of radius $\delta$ whose mutual distance is greater than or equal to $2 \varepsilon$. Under this assumption we have compared our model with Chan-Vese model, summarizing the results in Table 1.

Here we observe that the same heuristic computations can be repeated assuming that the the mutual distance is greater than some $\tilde{\varepsilon}$ with $\delta<\tilde{\varepsilon} \leq \varepsilon$. In this case we obtain the new link $1 / \lambda_{c v}=c \tilde{\varepsilon} / \sqrt{\lambda}$, and it turns out that the comparative analysis summarized in Table 1 still holds true in this case.

3. Approximated models for numerical computation. In this section we present our approximations of the proposed model (2.1) that will be used in our numerical experiments. 
3.1. The Lipschitz level set function approach. Here we propose a level set approach to our model, in the spirit of the successful method introduced by Chan-Vese [10] within the context of two-phase segmentation, following the level set formulation [24]. The main novelty of our method consists in the use of Lipschitz level set functions, with uniformly bounded Lipschitz constant (instead of $B V$ level set functions).

We recall that the main idea in [10] is to consider the gradient flow of the following functional:

$$
\begin{aligned}
\mathcal{H}_{c v}(\phi)= & \int_{\Omega}|\nabla H(\phi)| d x+\lambda\left[\int_{\Omega}\left|u_{0}-c_{1}(H(\phi))\right|^{2} H(\phi)\right) d x \\
& \left.+\int_{\Omega}\left|u_{0}-c_{2}(H(\phi))\right|^{2}(1-H(\phi)) d x\right],
\end{aligned}
$$

where $H$ is the Heaviside function defined by $H(z)=1$ for $z \geq 0$ and $H(z)=0$ otherwise, $\phi$ is the so-called level set function, with $\{\phi=0\}$ representing the unknown interface, and $c_{1}(H(\phi)), c_{2}(H(\phi))$ are defined as in (2.7) with $v$ replaced by $H(\phi)$.

One of the benefits of using the level set method is its efficiency even if initial conditions are chosen far from the original objects. Moreover, topological changes are automatically taken care of during the evolution of the level set function.

We are in a position to introduce the level set approximation of our model, working with the general formulation introduced in (2.12). Suppose $\phi$ be the signed distance function from the zero level set $\Gamma$. Thus, we have

$$
f\left(\frac{\operatorname{dist}(\cdot, \Gamma))}{\varepsilon}\right)=f\left(\frac{|\phi|}{\varepsilon}\right) .
$$

In order to force $\phi$ to look like a signed distance function, we add the penalization

$$
\frac{1}{p} \int_{\Omega}|\nabla \phi|^{p} d x
$$

This term, as $p \rightarrow \infty$, enforces $|\nabla \phi| \leq 1$, and in combination with the fitting term and the perimeter penalization ensures that any minimizing $\phi$ stays as a signed distance function from $\Gamma$. Other penalizations could be considered for this purpose, as for instance $p \int_{\Omega}(|\nabla \phi|-1)^{2} d x$. The advantage of our penalization lies in the fact that it is convex and the corresponding Euler-Lagrange equations involve the well-known p-Laplacian.

Summarizing, we propose the following functional to be minimized:

$$
\begin{aligned}
\mathcal{H}^{p}(\phi)= & \int_{\Omega} f\left(\frac{|\phi|}{\varepsilon}\right)+\frac{1}{p} \int_{\Omega}|\nabla \phi|^{p} d x+\lambda\left[\int_{\Omega}\left|u_{0}-c_{1}(H(\phi))\right|^{2} H(\phi) d x\right. \\
& \left.+\int_{\Omega}\left|u_{0}-c_{2}(H(\phi))\right|^{2}(1-H(\phi)) d x\right],
\end{aligned}
$$

where $c_{1}(H(\phi))$ and $c_{2}(H(\phi))$ are defined as for (3.1).

In order to show the relation between (3.3) and (2.12), it is convenient to rewrite the functional $\mathcal{H}^{p}$ in the following equivalent way. We define

$$
\begin{aligned}
& \widetilde{\mathcal{H}}^{p}: L^{\infty}(\Omega ;[0,1]) \times L^{\infty}(\Omega ;[-\operatorname{diam}(\Omega), \operatorname{diam}(\Omega)]) \rightarrow[0,+\infty] \\
&(v, \phi) \mapsto \begin{cases}\mathcal{H}^{p}(\phi) & \text { if } v=H(\phi), \\
+\infty & \text { otherwise }\end{cases}
\end{aligned}
$$


In order to describe the $\Gamma$-limit of $\widetilde{\mathcal{H}}^{p}$, as $p \rightarrow \infty$, we introduce the functional $J_{f}$ : $L^{\infty}(\Omega ;[0,1]) \times L^{\infty}(\Omega ;[0, \operatorname{diam}(\Omega)]) \mapsto[0,+\infty]$ defined by

$$
J_{f}(v, \phi):=\int_{\Omega} f\left(\frac{\phi}{\varepsilon}\right) d x+\lambda \int_{\Omega}\left[v\left(u_{0}-c_{1}(v)\right)^{2}+(1-v)\left(u_{0}-c_{2}(v)\right)^{2}\right] d x .
$$

Note that when $\phi$ is the distance function $d$ from $\Gamma(v)$, then $J_{f}(v, d)=\mathcal{E}_{f}(v)$. Thus, observing that

$$
\operatorname{dist}(x, \Gamma(v))=\sup \left\{|\phi(x)|: \phi \in W^{1, \infty}(\Omega),|\nabla \phi| \leq 1 \text { a.e. in } \Omega, \Gamma(v) \subseteq \phi^{-1}(0)\right\},
$$

and taking into account the monotonicity of $f$, the following relation between $J_{f}$ and $\mathcal{E}_{f}$ is easily established:

$$
\mathcal{E}_{f}(v)=\min \left\{J_{f}(v, \phi): \phi \in W^{1, \infty}(\Omega),|\nabla \phi| \leq 1 \text { a.e. in } \Omega, \Gamma(v) \subseteq \phi^{-1}(0)\right\} .
$$

Let us introduce the $\Gamma$-limit

$$
\begin{aligned}
& \mathcal{H}: L^{\infty}(\Omega ;[0,1]) \times L^{\infty}(\Omega ;[-\operatorname{diam}(\Omega), \operatorname{diam}(\Omega)]) \rightarrow[0,+\infty] \\
& (v, \phi) \mapsto \begin{cases}J_{f}(v,|\phi|) & \text { if } \phi \in W^{1, \infty}(\Omega),|\nabla \phi| \leq 1 \text { a.e. in } \Omega, \\
+\infty & v=0 \text { in }\{\phi<0\} \text { and } v=1 \text { in }\{\phi>0\} ;\end{cases}
\end{aligned}
$$

where $J_{f}$ is defined in (3.5).

We endow the space $L^{\infty}(\Omega ;[0,1]) \times L^{\infty}(\Omega ;[-\operatorname{diam}(\Omega)$, diam $(\Omega)])$ with the following metrizable convergence $\tau$

$$
\left(v_{n}, \phi_{n}\right) \stackrel{\tau}{\rightarrow}(v, \phi) \text { if and only if }\left\{\begin{array}{lll}
v_{n} \stackrel{*}{\rightarrow} v & \text { weakly* in } L^{\infty}(\Omega ;[0,1]), \\
\phi_{n} \rightarrow \phi & \text { uniformly in } \Omega .
\end{array}\right.
$$

We are ready to state the $\Gamma$-convergence result.

THEOREM 3.1. The functionals $\widetilde{\mathcal{H}}^{p}$ defined in (3.4) are equicoercive and $\Gamma$ converge, as $p \rightarrow \infty$, to the functional $\mathcal{H}$ defined in (3.8) with respect to the $\tau$ convergence (3.9).

Proof. First of all we prove the equicoercivity property. Let $p_{n} \rightarrow \infty$, and let $\left(v_{n}, \phi_{n}\right)$ be such that $\tilde{\mathcal{H}}_{f}^{p_{n}}\left(v_{n}, \phi_{n}\right) \leq C<+\infty$. Then, in particular, $\frac{1}{p_{n}} \int_{\Omega}\left|\nabla \phi_{n}\right|^{p_{n}} \leq$ $C$, and thus

$$
\left\|\nabla \phi_{n}\right\|_{p_{n}} \leq C^{\frac{1}{p_{n}}} p_{n}^{\frac{1}{p_{n}}} \rightarrow 1 \quad \text { as } n \rightarrow \infty .
$$

By the monotonicity of $p \mapsto\|\cdot\|_{p}$, it follows that the functions $\phi_{n}$ are uniformly bounded in $W^{1, p}$ for every fixed $p>1$. We conclude by Sobolev embedding that the sequence $\phi_{n}$ is precompact with respect to the uniform convergence. The precompactness of the sequence $v_{n}$ with respect to the weak* convergence follows immediately from the bound $0 \leq v_{n} \leq 1$.

Let us pass to the proof of the $\Gamma$-liminf inequality. let $\left(v_{n}, \phi_{n}\right) \stackrel{\tau}{\rightarrow}(v, \phi)$ and assume, without loss of generality, that $\tilde{\mathcal{H}}_{f}^{p_{n}}\left(v_{n}, \phi_{n}\right)$ is uniformly bounded. Note that the first integral in (3.5) is lower semicontinuous with respect to the uniform convergence in $\phi$, while the second one is continuous with respect to the weak* convergence 
in $v$, thanks also to (2.7). Hence, the $\Gamma$-liminf inequality will be proven once we show that $|\nabla \phi| \leq 1$ a.e. in $\Omega, v=0$ a.e. in $\{\phi<0\}$, and $v=1$ a.e. in $\{\phi>0\}$.

In order to check that $|\nabla \phi| \leq 1$ a.e. in $\Omega$, fix $q>1$, and observe that by semicontinuity

$$
\|\nabla \phi\|_{q} \leq \liminf _{n}\left\|\nabla \phi_{n}\right\|_{q} \leq \liminf _{n}\left\|\nabla \phi_{n}\right\|_{p_{n}}=1
$$

where we used the monotonicity of $p \mapsto\|\cdot\|_{p}$ and (3.10). Letting $q \rightarrow \infty$ in the above inequality we deduce that $\|\nabla \phi\|_{\infty} \leq 1$. The constraint $v=1$ in $\{\phi>0\}$ is a consequence of the fact that for every fixed $\delta>0, \phi_{n}>0$ in $\{\phi>\delta\}$ for $n$ large enough, so that $v_{n}$ and in turn $v$ are equal to 1 on $\{\phi \geq \delta\}$. Analogously we deduce $v=0$ in $\{\phi<0\}$.

In order to prove the $\Gamma$-limsup inequality let $p_{n} \rightarrow \infty$ and fix $(v, \phi)$ such that $\mathcal{H}(v, \phi)=J_{f}(v,|\phi|)<+\infty$. We may also assume $\Gamma(v) \neq \emptyset$ since otherwise the construction is trivial. Let $\left\{A_{n}\right\}$ be a sequence of open sets such that $A_{n} \subset \subset \frac{1}{n}-\Gamma(v)$ and $\chi_{A_{n}} \stackrel{*}{*} v \chi_{\Gamma(v)}$ and define

$$
v_{n}:= \begin{cases}0 & \text { in } A^{0} \backslash \overline{\frac{1}{n}-\Gamma(v)} \\ 1 & \text { in } A^{1} \backslash \overline{\frac{1}{n}-\Gamma(v)} \\ \chi_{A_{n}} & \text { otherwise }\end{cases}
$$

where $A^{0}$ and $A^{1}$ denote as usual the interior of $\tilde{v}^{-1}(0)$ and $\tilde{v}^{-1}(1)$, respectively. Finally, setting $d_{n}:=\operatorname{dist}\left(\cdot, \partial\left\{v_{n}=0\right\} \cap \Omega\right)$, we define

$$
\phi_{n}:=\left[\left(v_{n} d_{n}\right) \wedge \phi\right] \vee \frac{v_{n} d_{n}}{n}+\left[\left(\left(v_{n}-1\right) d_{n}\right) \vee \phi\right] \wedge \frac{\left(v_{n}-1\right) d_{n}}{n} .
$$

By construction $\phi_{n} \in W^{1, \infty}(\Omega)$ and $\left|\nabla \phi_{n}\right| \leq 1$ almost everywhere. Moreover, since on the open set $\left\{v_{n}=1\right\}$ we have $d_{n}>0$, it follows from the definition of $\phi_{n}$ that $\left\{v_{n}=1\right\} \subseteq\left\{\phi_{n}>d_{n} / n>0\right\}$. On the other hand, again from the definition of $\phi_{n}$ we have $\left\{v_{n}=0\right\} \subseteq\left\{\phi_{n} \leq 0\right\}$. Hence, necessarily $\left\{v_{n}=1\right\}=\left\{\phi_{n}>0\right\}$ and $\left\{v_{n}=0\right\}=\left\{\phi_{n} \leq 0\right\}$ and thus

$$
v_{n}=H\left(\phi_{n}\right) .
$$

We now show that $\phi_{n} \rightarrow \phi$ uniformly. As $\partial\left\{v_{n}=0\right\} \cap \Omega \subseteq \overline{\frac{1}{n}-\Gamma(v)} \cap \Omega$, we may assume (passing to a subsequence if necessary) that $\partial\left\{v_{n}\right\} \rightarrow \Gamma \subseteq \overline{\Gamma(v)}$ in the Hausdorff convergence. Hence, setting $d:=\operatorname{dist}(\cdot, \Gamma)$, we have

$$
d \geq \operatorname{dist}(\cdot, \Gamma(v))
$$

and $d_{n} \rightarrow d$ uniformly. In turn, since $v_{n} \stackrel{*}{*} v$ and $\left\{v_{n} d_{n}\right\}$ is still a sequence of bounded equi-Lipschitz functions, we have $v_{n} d_{n} \rightarrow v d$ uniformly. We conclude

$$
\phi_{n} \rightarrow \psi:=[(v d) \wedge \phi] \vee 0+[((v-1) d) \vee \phi] \wedge 0 \quad \text { uniformly. }
$$

On $\{\phi>0\}$ we have $v=1$ and, in turn, $\psi=d \wedge \phi=\phi$, where the last equality follows from (3.7) and (3.11). Analogously, on $\{\phi<0\}$ we have $v=0$ and, in turn, $\psi=-d \vee \phi=\phi$. Moreover, from the very definition of $\psi, \psi=0$ on $\{\phi=0\}$. Summarizing, $\psi=\phi$ and $\left(v_{n}, \phi_{n}\right) \stackrel{\tau}{\rightarrow}(v, \phi)$. Therefore, we have

$$
\int_{\Omega} f\left(\frac{\phi_{n}}{\varepsilon}\right) d x+\lambda \int_{\Omega}\left[v_{n}\left(u_{0}-c_{1}\left(v_{n}\right)\right)^{2}+\left(1-v_{n}\right)\left(u_{0}-c_{2}\left(v_{n}\right)\right)^{2}\right] d x \rightarrow \tilde{\mathcal{H}}_{f}(v, d) .
$$


Since $\left\|\nabla \phi_{n}\right\| \leq 1$ a.e., also $\frac{1}{p_{n}} \int_{\Omega}\left|\nabla \phi_{n}\right|^{p_{n}} d x \rightarrow 0$ as $n \rightarrow \infty$. This establishes the $\Gamma$-limsup inequality.

Since the $\Gamma$-convergence of equicoercive functionals implies the convergence of minimizers (we refer the reader to [5], [15]), taking into account (3.4), (3.6), and (3.7), we have the following corollary which clarifies the relation between (3.3) and (2.12).

CoRollary 3.2. Let $p_{n} \rightarrow+\infty$ and let $\phi_{n} \in L^{\infty}(\Omega ;[-\operatorname{diam}(\Omega)$, $\operatorname{diam}(\Omega)])$ be a minimizer for the functional $\mathcal{H}^{p_{n}}$ defined in (3.3). Then, up to a subsequence, $\left(H\left(\phi_{n}\right), \phi_{n}\right) \stackrel{\tau}{\rightarrow}(v, \phi)$ with $v$ a minimizer for $\mathcal{E}_{f},|\phi|=\operatorname{dist}(\cdot, \Gamma(v)), v=0$ on $\{\phi<0\}$, and $v=1$ on $\{\phi>0\}$.

REMARK 9 (Relation with the Chan-Vese model). Consider a one-parameter family $\left(H_{\rho}\right)_{\rho>0}$ of smeared versions of the Heaviside function, where the sharp discontinuity is replaced by a continuous transition on an interval of order $\rho$. More precisely, we let $h: \mathbb{R} \rightarrow[0,1]$ be any Lipschitz continuous non-decreasing function satisfying

$$
\lim _{t \rightarrow-\infty} h(t)=0, \quad \lim _{t \rightarrow+\infty} h(t)=1, \quad \text { and }
$$

$h^{\prime}$ is an even l.s.c. function, non-increasing in $[0,+\infty)$,

and for all $t \in \mathbb{R}$ we set

$$
H_{\rho}(t):=h\left(\frac{t}{\rho}\right) .
$$

In the Chan-Vese model, the regularization term $\int_{\Omega}|\nabla H(\phi)| d x$ is approximated by

$$
\int_{\Omega}\left|\nabla H_{\rho}(\phi)\right| d x=\int_{\Omega}\left|H_{\rho}^{\prime}(\phi)\right||\nabla \phi| d x,
$$

which is precisely the weighted total variation of $\phi$ concentrated at its zero level set.

In our approach we think of $\phi$ as a signed distance function, so that $|\nabla \phi|=1$. Hence, we replace the above regularization term by $\int_{\Omega}\left|H_{\varepsilon}^{\prime}(\phi)\right| d x$ and the approximation of $\mathcal{L}^{2}(\varepsilon-\Gamma)$ looks like

$$
\int_{\Omega} f\left(\frac{|\phi|}{\varepsilon}\right) d x=\varepsilon \int_{\Omega}\left|H_{\varepsilon}^{\prime}(\phi)\right| d x=\int_{\Omega} h^{\prime}\left(\frac{\phi}{\varepsilon}\right) d x .
$$

The presence of the gradient penalization in (3.2) in the proposed model (3.3) enforces $\phi$ to be the signed distance function and represents the main difference with the classical Chan-Vese approach (3.1).

REMARK 10. Note the appearance of $\varepsilon$ in front of the perimeter penalization in (3.13). This is mainly motivated by notational consistency with (2.12) (see also Remark 2). Of course there is no loss of generality in writing the functional in this way, since $\varepsilon$ and $\lambda$ are independent parameters.

One example of smooth function $h$ that satisfies (3.12) is given by

$$
h(x):=\frac{1}{\sqrt{\pi}} \int_{-\infty}^{x} e^{-t^{2}} d t,
$$

so that the nonlocal perimeter penalization becomes

$$
\varepsilon \int_{\Omega}\left|H_{\varepsilon}^{\prime}(\phi)\right| d x=\int_{\Omega} h^{\prime}\left(\frac{\phi}{\varepsilon}\right) d x=\frac{1}{\sqrt{\pi}} \int_{\Omega} e^{-\frac{\phi^{2}}{\varepsilon^{2}}} d x .
$$


Note that here we normalize $h$ so that $\int_{\mathbb{R}} h(x) d x=1$, but this normalization is indeed unnecessary. Other examples of $h$ such that $h^{\prime}\left(\frac{x}{\varepsilon}\right)$ better approximate the characteristic function of the interval $[-1,1]$ include $h_{k}(x)=\int_{-\infty}^{x} e^{-t^{k}} d t$, for large even $k$. In other words, we have

$$
\mathcal{L}^{2}(\varepsilon-\Gamma) \approx \int_{\Omega} e^{-\frac{\phi(x)^{k}}{\varepsilon^{k}}} d x
$$

Such a function will be used in the numerical computations presented in the next section.

In order to approximate a local minimizer of $\mathcal{H}^{p}$, in our simulations we will introduce an artificial time $t$ and project to the gradient descent direction, thus solving

$$
\frac{\partial \phi}{\partial t}=\frac{k}{\varepsilon^{k}} \phi^{k-1} e^{-(\phi / \varepsilon)^{k}}+\operatorname{div}\left(|\nabla \phi|^{p-2} \nabla \phi\right)-\lambda \delta(\phi)\left[\left(u_{0}-c_{1}\right)^{2}-\left(u_{0}-c_{2}\right)^{2}\right]
$$

and updating the constants $c_{1}$ and $c_{2}$ at each time step according to the formulas

$$
c_{1}=\frac{\int_{\Omega} H(\phi) u_{0} d x}{\int_{\Omega} H(\phi) d x} \quad \text { and } \quad c_{2}=\frac{\int_{\Omega}(1-H(\phi)) u_{0} d x}{\int_{\Omega}(1-H(\phi)) d x} .
$$

Here, $\delta(\phi)$ represents the Dirac delta function centered at 0 . Numerically, we approximate $\delta(\phi)$ by

$$
\delta_{\gamma}(\phi)=\frac{1}{1+(\phi / \gamma)^{2}}, \text { for some small } \gamma>0 .
$$

3.2. Ambrosio-Tortorelli type approximation. Here we discuss a phase field approximation of our proposed functional, based on classical Ambrosio-Tortorelli approximation of free discontinuity problems (see [1]). The main feature of such approximation is that it allows for discontinuity sets that are not boundary of some other set. Even if we do not need to treat such general discontinuity sets, we aim to show that also in this context it can be easily obtained a diffused transition region, enforcing some Lipschitz regularity on the phase-field function. We believe indeed that such approach could be useful also in other contexts, as in fracture mechanics, where recently a model for smeared crack set has been proposed by B. Schmidt, F. Fraternali, and M. Ortiz. in [14].

We will work with the general formulation of our model introduced in (2.12). We now introduce the approximating functionals. For $n \in \mathbb{N}$ and $p>1$ let $J_{f}^{n, p}$ : $L^{\infty}(\Omega ;[0,1]) \times L^{\infty}(\Omega,[0, \operatorname{diam}(\Omega)]) \mapsto[0,+\infty]$ be defined by

$$
\begin{aligned}
J_{f}^{n, p}(v, d)= & \int_{\Omega} f\left(\frac{d}{\varepsilon}\right) d x+\lambda \int_{\Omega}\left[v\left(u_{0}-c_{1}(v)\right)^{2}+(1-v)\left(u_{0}-c_{2}(v)\right)^{2}\right] d x \\
& +n \int_{\Omega} v^{2}(1-v)^{2} d x+n \int_{\Omega} d^{2}|\nabla v|^{2} d x+\frac{1}{p} \int_{\Omega}|\nabla d|^{p} d x
\end{aligned}
$$

if $v \in W^{1,2}(\Omega)$ and $d \in W^{1, p}(\Omega)$, and $+\infty$ otherwise. Here $f$ is as in Remark 5 a positive non increasing lower semicontinuous summable function and $c_{1}(v), c_{2}(v)$ are as in (2.7).

The heuristics behind such approximation is the following: for $n$ and $p$ tending to infinity, the last three terms in (3.14) force the segmentation $v$ to be a characteristic function, $\Gamma(v)$ to coincide with the level set $\{d=0\}$, and $d$ to approximate the distance 
function from $\Gamma(v)$, respectively. In turn, the first two addends will approximate the perimeter penalization and the fidelity term, respectively.

The main difference between the level set and the Ambrosio Tortorelli approximation is that here $v$ and the distance function are independent variables and the coupling $\Gamma(v) \subseteq\{d=0\}$ is enforced only in the limit, while in the definition of $\widetilde{\mathcal{H}}^{p}$ in (3.4), $v$ and the signed function $\phi$ are coupled by the relation $v=H(\phi)$.

In order to state the $\Gamma$-convergence result for $J_{f}^{n, p}$, we introduce the functional $J_{f}: L^{\infty}(\Omega ;[0,1]) \times L^{\infty}(\Omega ;[0, \operatorname{diam}(\Omega)]) \rightarrow[0,+\infty]$ defined by

$$
J_{f}(v, d):=\int_{\Omega} f\left(\frac{d}{\varepsilon}\right) d x+\lambda \int_{\Omega}\left[v\left(u_{0}-c_{1}(v)\right)^{2}+(1-v)\left(u_{0}-c_{2}(v)\right)^{2}\right] d x,
$$

Note that when $d$ is the distance function from $\Gamma(v)$, then $J_{f}(v, d)=\mathcal{E}_{f}(v)$. Thus, observing that

$$
\operatorname{dist}(x, \Gamma(v))=\sup \left\{d(x): d \in W^{1, \infty}(\Omega),|\nabla d| \leq 1 \text { a.e. in } \Omega, \Gamma(v) \subseteq d^{-1}(0)\right\},
$$

and taking into account the monotonicity of $f$, the following relation between $J_{f}$ and $\mathcal{E}_{f}$ is easily established:

$$
\mathcal{E}_{f}(v)=\min \left\{J_{f}(v, d): d \in W^{1, \infty}(\Omega),|\nabla d| \leq 1 \text { a.e. in } \Omega, \Gamma(v) \subseteq d^{-1}(0)\right\} .
$$

We endow the space $L^{\infty}(\Omega ;[0,1]) \times L^{\infty}(\Omega ;[0, \operatorname{diam}(\Omega)])$ with the following (metrizable) convergence $\tau$ :

$$
\left(v_{n}, d_{n}\right) \stackrel{\tau}{\rightarrow}(v, d) \text { if and only if } \begin{cases}v_{n} * * & \text { weakly* in } L^{\infty}(\Omega ;[0,1]) \\ d_{n} \rightarrow d & \text { uniformly in } \Omega .\end{cases}
$$

We are in a position to state the $\Gamma$-convergence theorem.

TheOrEm 3.3. The functionals $J_{f}^{n, p}$ defined in (3.14) are equicoercive and $\Gamma$ converge (as $n, p \rightarrow+\infty$, with respect to the $\tau$-convergence defined in (3.17)) to the functional $G_{f}(v, d)$ defined as $J_{f}(v, d)$ if $|\nabla d| \leq 1$ a.e. in $\Omega$ and $\Gamma(v) \subseteq d^{-1}(0)$, and $+\infty$ otherwise.

Proof. First of all we prove the equicoercivity property. Let $p_{n} \rightarrow \infty$, and let $\left(v_{n}, d_{n}\right)$ be such that $J_{f}^{n, p_{n}}\left(v_{n}, d_{n}\right) \leq C<+\infty$. Then, in particular, $\frac{1}{p_{n}} \int_{\Omega}\left|\nabla d_{n}\right|^{p_{n}} \leq$ $C$, and thus

$$
\left\|\nabla d_{n}\right\|_{p_{n}} \leq C^{\frac{1}{p_{n}}} p_{n}^{\frac{1}{p_{n}}} \rightarrow 1 \quad \text { as } n \rightarrow \infty .
$$

By the monotonicity of $p \mapsto\|\cdot\|_{p}$, it follows that the functions $d_{n}$ are uniformly bounded in $W^{1, p}$ for every fixed $p>1$. We conclude by Sobolev embedding that the sequence $d_{n}$ is precompact with respect to the uniform convergence. The precompactness of the sequence $v_{n}$ with respect to the weak ${ }^{*}$ convergence follows immediately from the bound $0 \leq v_{n} \leq 1$.

We now prove the $\Gamma$-convergence result. In order to establish the $\Gamma$-liminf inequality, let $\left(v_{n}, d_{n}\right) \stackrel{\tau}{\rightarrow}(v, d)$ and assume, without loss of generality, that $J_{f}^{n, p_{n}}\left(v_{n}, d_{n}\right)$ is uniformly bounded.

Note that the first integral in (3.14) is lower semicontinuous with respect to the uniform convergence in $d$, while the second one is continuous with respect to the weak* convergence in $v$, thanks also to (2.7). Hence, since the remaining terms in (3.14) are positive, the $\Gamma$-liminf inequality will be proven once we show that $|\nabla d| \leq 1$ a.e. in $\Omega$ and $\Gamma(v) \subseteq d^{-1}(0)$. 
In order to check that $|\nabla d| \leq 1$ a.e. in $\Omega$, fix $q>1$, and observe that by semicontinuity

$$
\|\nabla d\|_{q} \leq \liminf _{n}\left\|\nabla d_{n}\right\|_{q} \leq \liminf _{n}\left\|\nabla d_{n}\right\|_{p_{n}}=1
$$

where we used the monotonicity of $p \mapsto\|\cdot\|_{p}$ and (3.18). Letting $q \rightarrow \infty$ in the above inequality we deduce that $\|\nabla d\|_{\infty} \leq 1$.

In order to show that $\Gamma(v) \subseteq d^{-1}(0)$, let $\Omega^{+}:=\{x \in \Omega: d(x)>0\}$. By uniform convergence, on any compact subset $K \subset \Omega^{+}$we have $\lim _{n} \min _{K} d_{n}=\min _{K} d>0$. Then, by the fact that $n \int_{\Omega}^{+} d_{n}^{2}\left|\nabla v_{n}\right|^{2} d x$ is uniformly bounded it follows that $v_{n} \rightarrow v$ strongly in $W_{l o c}^{1,2}\left(\Omega^{+}\right)$and $\nabla v=0$ in $\Omega^{+}$. In turn, since $n \int_{\Omega}^{+} v_{n}^{2}\left(1-v_{n}\right)^{2} d x$ is uniformly bounded, we deduce that $v$ restricted to $\Omega^{+}$is a characteristic function, constant on each connected component of $\Omega^{+}$. In particular, $\Omega^{+}$is contained in the union of the interior of $v^{-1}(0)$ and the interior of $v^{-1}(1)$, and hence $\Gamma(v) \subseteq \Omega \backslash \Omega^{+}=$ $d^{-1}(0)$. As observed before, this establishes the $\Gamma$-liminf inequality.

Let us pass now to the proof of the $\Gamma$-limsup inequality. Let $(v, d)$ be such that $G_{f}(v, d)<+\infty$ and consider the sequence $v_{n}$ defined as in (2.8). Then, with an argument similar to that used in the proof of Proposition 2.3, we have $J_{f}\left(v_{n}, d\right) \rightarrow J_{f}(v, d)$. This observation, combined with a standard diagonalization procedure, shows that it is enough to prove the $\Gamma$-limsup inequality assuming that $v$ is a characteristic function. Hence, fix $(v, d)$ with $v$ a characteristic function and $J_{f}(v, d)<+\infty$, let $h_{n} \rightarrow \infty$ and $\rho_{n}$ be a sequence of standard mollifiers with support in $B_{1 / h_{n}}(0)$. We set

$$
v_{n}:=\rho_{n} * v, \quad d_{n}(x):=\operatorname{dist}\left(x, \frac{2}{h_{n}}-\Gamma(v)\right) \wedge d .
$$

Then we clearly have that $v_{n} \rightarrow v$ strongly in $L^{1}$ and $d_{n} \rightarrow d$ uniformly, so that

$$
\int_{\Omega} f\left(\frac{d_{n}}{\varepsilon}\right) d x+\lambda \int_{\Omega}\left[v_{n}\left(u_{0}-c_{1}\left(v_{n}\right)\right)^{2}+\left(1-v_{n}\right)\left(u_{0}-c_{2}\left(v_{n}\right)\right)^{2}\right] d x \rightarrow J_{f}(v, d) .
$$

Moreover, by construction $\operatorname{supp} \nabla v_{n} \subseteq\left\{d_{n}=0\right\}$, and thus $n \int_{\Omega} d_{n}^{2}\left|\nabla v_{n}\right|^{2} d x=0$. Since $\left\|\nabla d_{n}\right\| \leq 1$ a.e., also $\frac{1}{p_{n}} \int_{\Omega}\left|\nabla d_{n}\right|^{p_{n}} d x \rightarrow 0$ as $n \rightarrow \infty$. Finally, we can suitably choose $h_{n}$ going to infinity so fast that $n \int_{\Omega} v_{n}^{2}\left(1-v_{n}\right)^{2} d x \rightarrow 0$, and this establishes also the $\Gamma$-limsup inequality.

Since the $\Gamma$-convergence of equicoercive functionals implies the convergence of minimizers, taking into account (3.15) and (3.16), we have the following immediate corollary of the previous theorem.

Corollary 3.4. Let $p_{n} \rightarrow+\infty$ and for every $n \in \mathbb{N}$ let $\left(v_{n}, d_{n}\right)$ be a minimizer for $J_{f}^{n, p_{n}}$. Then, up to a subsequence, $\left(v_{n}, d_{n}\right) \stackrel{\tau}{\rightarrow}(v, d)$ with $d=\operatorname{dist}(\cdot, \Gamma(v))$ and $v$ a minimizer for $\mathcal{E}_{f}$.

In order to minimize the functional $J_{f}^{n, p}$ with respect to each of its variable, in our numerical experiments we project to the gradient descent direction and, by introducing an artificial time $t$, we solve the following time dependent system of equations

$$
\begin{aligned}
v_{t}= & =-\lambda\left[\left(u_{0}-c_{1}\right)^{2}-\left(u_{0}-c_{2}\right)^{2}\right] \\
& -2 n\left[v(1-v)^{2}-v^{2}(1-v)+\operatorname{div}\left(d^{2}|\nabla v|\right)\right], \\
d_{t}= & =k \frac{d^{k-1}}{\varepsilon^{k}} e^{-\frac{d^{k}}{\varepsilon^{k}}}-2 n d|\nabla v|^{2}+\operatorname{div}\left(|\nabla \phi|^{p-2} \nabla \phi\right) .
\end{aligned}
$$


At each time step we update the constants $c_{1}$ and $c_{2}$ according to the formulas

$$
c_{1}=\frac{\int_{\Omega} v u_{0} d x}{\int_{\Omega} v d x} \quad \text { and } \quad c_{2}=\frac{\int_{\Omega}(1-v) u_{0} d x}{\int_{\Omega}(1-v) d x} .
$$

3.3. The infinite Laplacian to select the length-scale $\varepsilon$. Here we propose a variant of our model in the attempt of automatically select the length-scale $\varepsilon$ through a minimization procedure. To this end, consider our proposed functional $\mathcal{E}_{f}$ defined in $(2.12)$ where $\varepsilon$ in not anymore a parameter, but a variable of the functional. We clearly have that such an energy is minimized for $\varepsilon \rightarrow 0$. For this reason, in order to have a non trivial variational problem we could think of adding a term penalizing too small $\varepsilon$. These considerations lead to the following basic definition

$\mathcal{E}_{f}(v, \varepsilon):=\int_{\Omega} f\left(\frac{\operatorname{dist}(x, \Gamma(v))}{\varepsilon}\right) d x+\lambda \min _{c_{1}, c_{2} \in \mathbb{R}} \int_{\Omega}\left[v\left(u_{0}-c_{1}\right)^{2}+(1-v)\left(u_{0}-c_{2}\right)^{2}\right] d x+\frac{1}{\varepsilon}$.

The main motivation to consider such a functional is that it leads indeed to very natural approximations, based on the use of the infinite laplacian. On the other hand, we stress out that at the present we don't have theoretical motivations or experimental evidence that such a variant of our model represents an efficient way of selecting the length-scale $\varepsilon$. Exploiting this point is not the purpose of this paper but of future interests, and would need a specific interesting analysis.

To approximate the energy functional (3.19) in the Lipschitz level set method we fix an $\varepsilon>0$, and replace the term $\frac{1}{p_{n}} \int_{\Omega}|\nabla \phi|^{p_{n}}$ by $\|\nabla \phi\|_{p}$, or equivalently with its $\Gamma$-limit $\|\nabla \phi\|_{\infty}$ (see [17]), obtaining in this way the following functional

$$
\begin{aligned}
\mathcal{H}^{\infty}(\phi)= & \int_{\Omega} f(|\phi|) d x+\|\nabla \phi\|_{L^{\infty}(\Omega)}+\lambda\left[\int_{\Omega}\left|u_{0}-c_{1}(H(\phi))\right|^{2} H(\phi) d x\right. \\
& \left.+\int_{\Omega}\left|u_{0}-c_{2}(H(\phi))\right|^{2}(1-H(\phi)) d x\right] .
\end{aligned}
$$

Analogously, we could consider also the Ambrosio-Tortorelli approximation, obtained by minimization of the following energy

$$
\begin{aligned}
J_{f}^{n, \infty}(v, d)= & \int_{\Omega} f(d) d x+\lambda \int_{\Omega}\left[v\left(u_{0}-c_{1}(v)\right)^{2}+(1-v)\left(u_{0}-c_{2}(v)\right)^{2}\right] d x \\
& +n \int_{\Omega} v^{2}(1-v)^{2} d x+n \int_{\Omega} d^{2}|\nabla v|^{2} d x+\|\nabla d\|_{L^{\infty}(\Omega)} .
\end{aligned}
$$

We refer the interest readers to Aronsson [2] and Aronsson-Crandall-Juutinen [3] and references therein for a thorough study of the problem of absolutely minimizing Lipschitz extension, and to [17] for the corresponding power law approximation. Here, we just mention that the corresponding Euler-Lagrange equation is the so-called infinite laplacian, defined by

$$
\Delta_{\infty} \phi=\frac{1}{|\nabla \phi|^{2}}\left[\phi_{x_{1}}^{2} \phi_{x_{1} x_{2}}+2 \phi_{x_{1}} d_{x_{2}} \phi_{x_{1} x_{2}}+\phi_{x_{2}}^{2} \phi_{x_{2} x_{2}}\right]=0 .
$$

The time dependent equation

$$
\frac{\partial \phi}{\partial t}=\Delta_{\infty} \phi, \text { and } \phi(0, x)=\phi_{0}(x)
$$




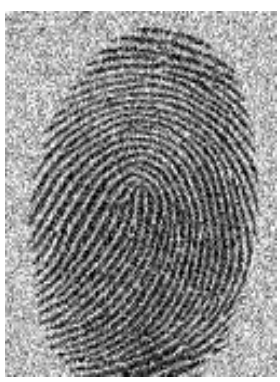

$u_{0}$

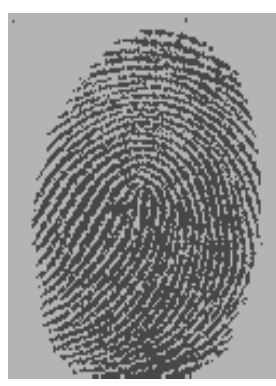

$u$

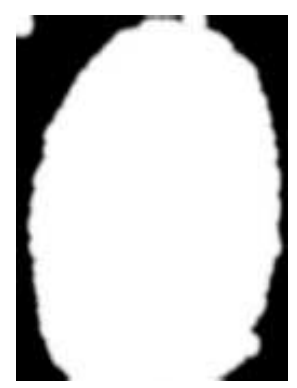

$\varepsilon-\Gamma(u)$

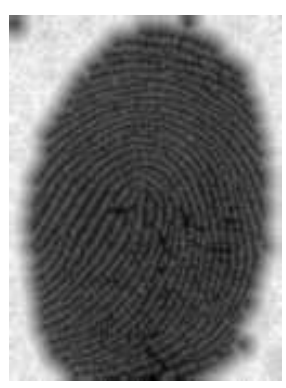

$\phi$

FIGURE 4.1. The segmented fingerprint using our presented model, approximated through the Lipschitz level set method, with $\lambda=20$ and $\varepsilon=40$.

with Neumann boundary condition has been applied to image interpolation by CasellesMorel-Sbert [9] and most recently by Elion-Vese [12] to image decomposition. In [23], Oberman proposed a convergent scheme for $\Delta_{\infty}$. It is this scheme with 4 -pointneighbor that we'll use for the numerical computations based on Lipschitz level set method, solving

$$
\frac{\partial \phi}{\partial t}=\frac{k}{\varepsilon^{k}} \phi^{k-1} e^{-(\phi / \varepsilon)^{k}}+\Delta_{\infty} \phi-\lambda \delta(\phi)\left[\left(u_{0}-c_{1}\right)^{2}-\left(u_{0}-c_{2}\right)^{2}\right]
$$

and updating at each time step the constants $c_{1}$ and $c_{2}$ according to the following formulas

$$
c_{1}=\frac{\int_{\Omega} H(\phi) u_{0} d x}{\int_{\Omega} H(\phi) d x} \quad \text { and } \quad c_{2}=\frac{\int_{\Omega}(1-H(\phi)) u_{0} d x}{\int_{\Omega}(1-H(\phi)) d x} .
$$

4. Numerical results. In this section we show some numerical results for our proposed model. We will focus on the level set approximation, based on the use of Lipschitz functions, comparing the results with those obtained implementing the classical Chan-Vese model [10].

We begin by showing the result of our model on a fingerprint with noise. In Figure 4.1 we see that our model is capable to preserve all the details of the fingerprint, removing almost all the noise from the picture. We fix $p=15$ and we approximate the $\varepsilon$-neighborhood of $\Gamma(u)$ with $f\left(\frac{\phi}{\varepsilon}\right):=e^{-(\phi / \varepsilon)^{8}}$. Moreover we chose $\lambda=20, \varepsilon=40$.

In the next experiment we will see the effect of tuning the parameter $\varepsilon$ on the segmented image. In Figure 4.2 we take $\lambda=20$, and $\varepsilon=10,20,30,40$. Increasing $\varepsilon$ we increase the penalization effect on the dilute noise, so that the denoising effect increases. For $\varepsilon=40$ we get an almost complete denoising.

On the other hand, our model predicts that near the boundaries of the finger print penalization effect is relaxed, and indeed increasing $\varepsilon$ does not affect the details of the finger print. This relaxation effect of our model can be understood looking at the $\varepsilon$-neighborhood of $\Gamma(u)$ : for $\varepsilon=20,30,40$, the set $\varepsilon-\Gamma(u)$ covers all the fingerprint, and therefore in this region the penalization of the boundaries is indeed independent of $\varepsilon$.

Toning $\lambda$ in our model clearly increases the amount of details in the pictures. We show this aspect in Figure 4.3, where we fix $\varepsilon=20$, and we chose $\lambda=10,50,500$. Increasing $\lambda$ we get more details of the branch of the three, while (for this specific 

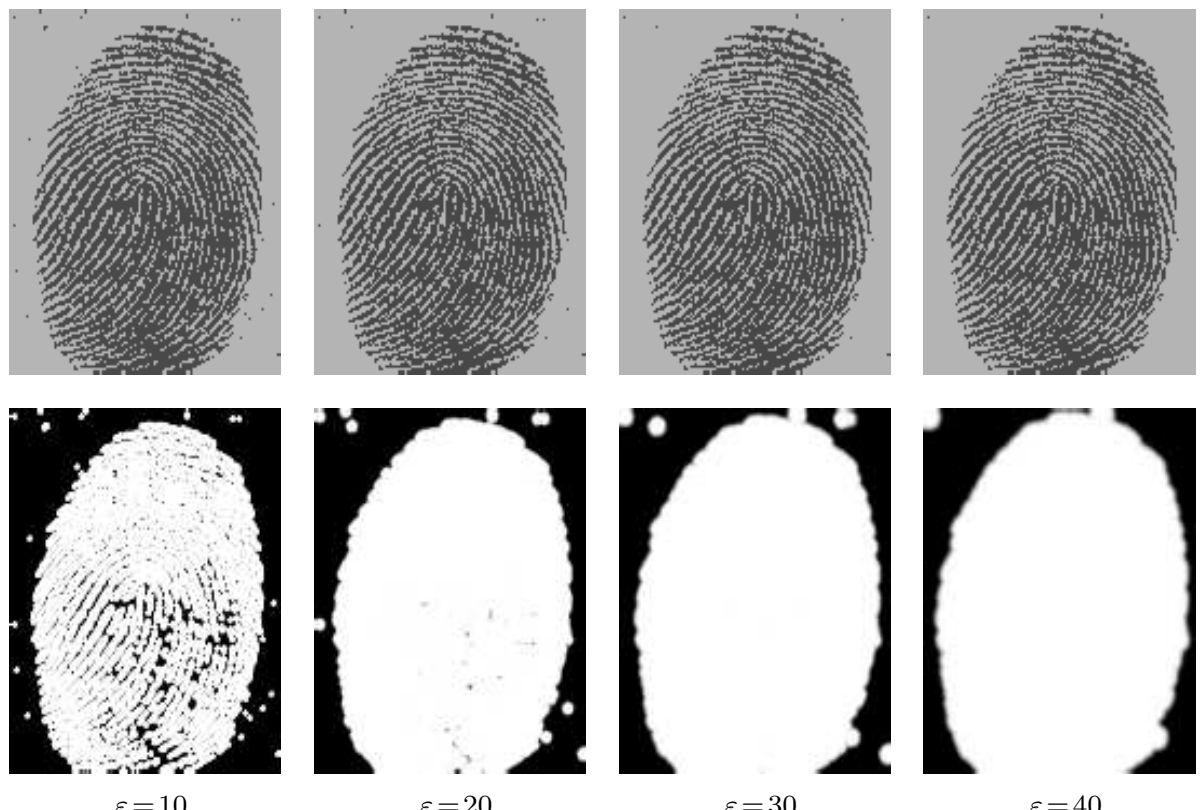

$\varepsilon=10$

$\varepsilon=20$

$\varepsilon=30$

$\varepsilon=40$

FIGURE 4.2. The segmented fingerprint $u$ and the $\varepsilon$-neighborhood of $\Gamma(u)$, approximated through the Lipschitz level set method, with $\lambda=20$ and $\varepsilon=10,20,30,40$.

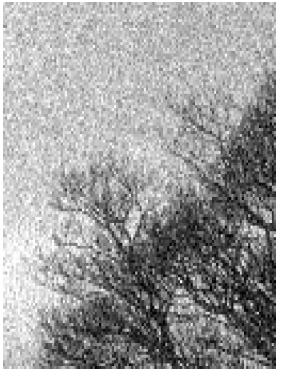

$u_{0}$

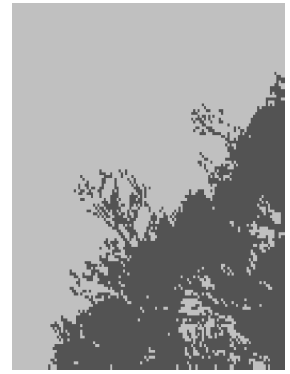

$u, \lambda=10$

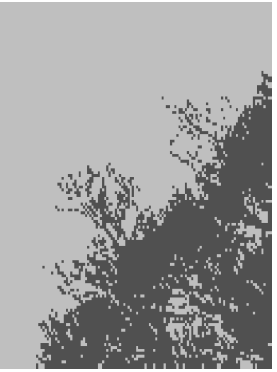

$u, \lambda=50$

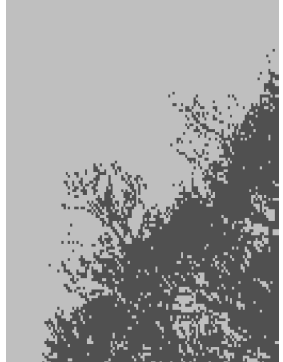

$u, \lambda=500$

Figure 4.3. The segmented tree with $\varepsilon=20$ and $\lambda=10,50,500$.

picture) the denoising is almost complete. Only for $\lambda=500$ some pixel assimilable to noise appears.

In order to compare our model with Chan-Vese model, we implement a picture representing a nebula, surrounded by stars. We consider here the stars as noise to be removed, while we want to identify the contours of the nebula. in Figure 4.4 we show the results with $\varepsilon=20$ and with $\lambda=80,100,120$, and we compare the results with the Chan-Vese model with $\lambda_{c v}=\lambda-60$. With this choice of the parameters, we have that our model exhibit slight finer details of the contour of the nebula, while the denoising effect if largely better.

The difference between the two models can be clearly understood looking at the level set functions, that are very sharp in the Chan-Vese model (approximating a characteristic $B V$ function), and diffused in our model (being a Lipschitz function).

In Figure 4.5 we compare the segmented nebula for very big $\lambda$ 's. We have that 


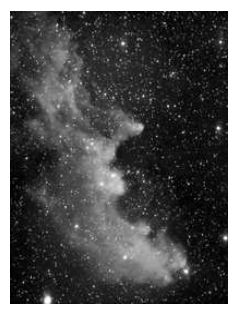

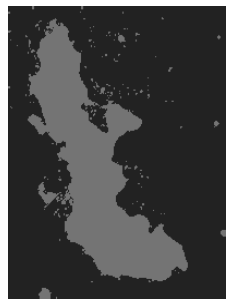

$u, \varepsilon=20, \lambda=80$

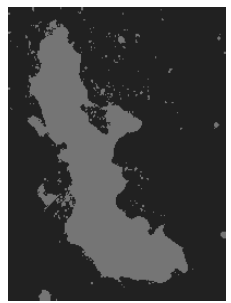

$u, \varepsilon=20, \lambda=100$

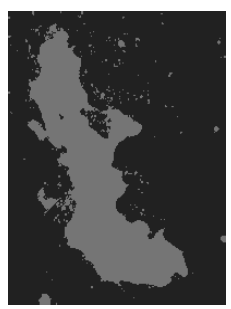

$u, \varepsilon=20, \lambda=120$

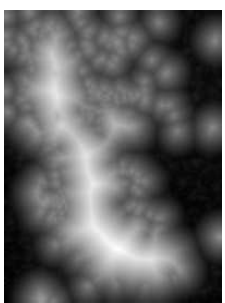

$\phi, \lambda=80$

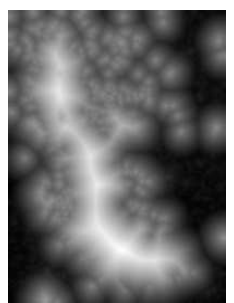

$\phi, \lambda=100$

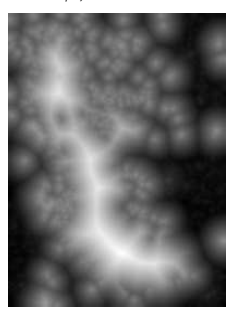

$\phi, \lambda=120$

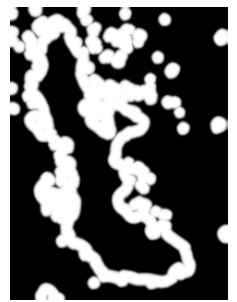

$\varepsilon-\Gamma(u), \lambda=80$

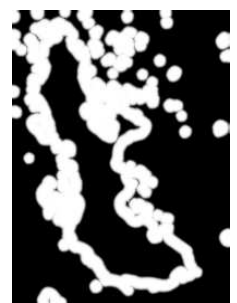

$\varepsilon-\Gamma(u), \lambda=100$

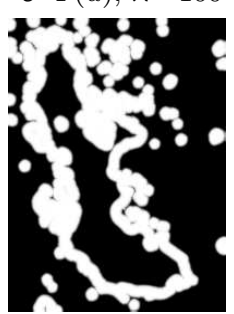

$\varepsilon-\Gamma(u), \quad \lambda=120$

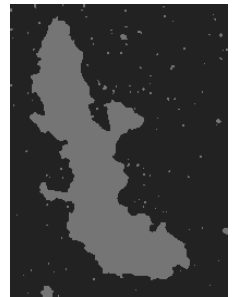

$u_{c v}, \lambda_{c v}=20$

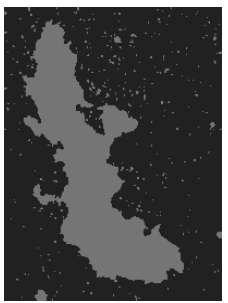

$u_{c v}, \lambda_{c v}=40$

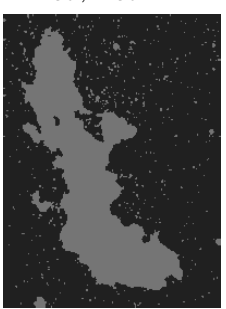

$u_{c v}, \lambda_{c v}=60$

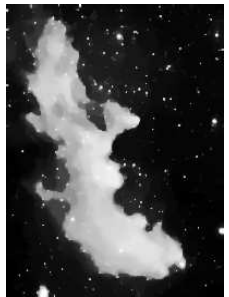

$\phi_{c v}, \lambda_{c v}=20$

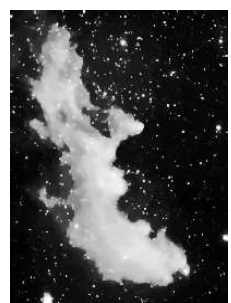

$\phi_{c v}, \lambda_{c v}=40$

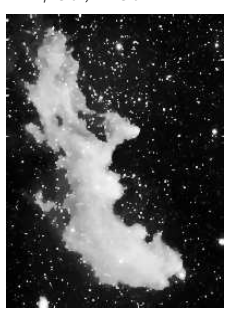

$\phi_{c v}, \lambda_{c v}=60$

Figure 4.4. Comparison between the proposed model and Chan-Vese model. Here we chose $\lambda_{c v}=\lambda-60$, for $\lambda=80,100,120$, and $\varepsilon=20$.

both model preserve very fine details of the nebula, while the denoising is very weak. The proposed model performs a little better.

In Figure 4.6 we show the effect of tuning the parameter $\varepsilon$ in the nebula: we fix $\lambda=50$, and we consider $\varepsilon=10,20,30,40$. We see that increasing $\varepsilon$, the denoising effect in the regions far from the nebula increases: isolated stars disappear increasing $\varepsilon$. At the same time, the details of the contour of the nebula increase. This effect is particularly evident on the top of the picture. On the other hand, increasing $\varepsilon$ increases also the number of stars around the contour of the nebula: toning $\varepsilon$ we determine the length-scale of the region were we want to keep fine oscillations of the boundary, and this region turns out to attract also some noise, according with Remark 6, assimilated to fine oscillations of the boundary of the segmented image. At 


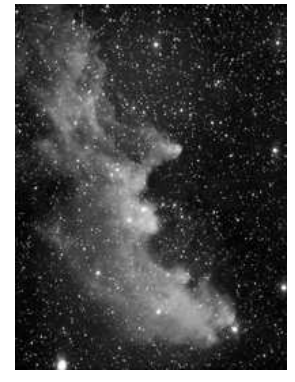

$u_{0}$

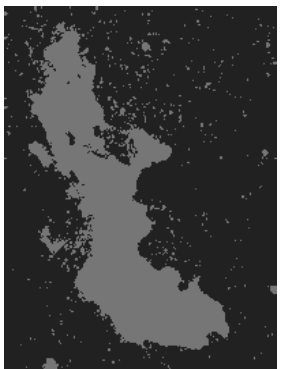

$u, \varepsilon=20, \lambda=200$

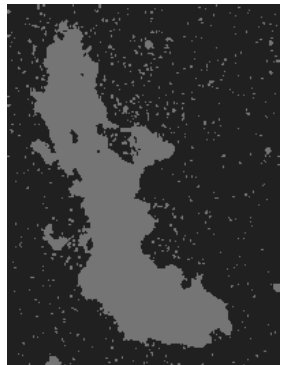

$u_{c v}, \lambda_{c v}=100$

FIGURE 4.5. The segmented image $u$ and $u_{c v}$ for big $\lambda$.
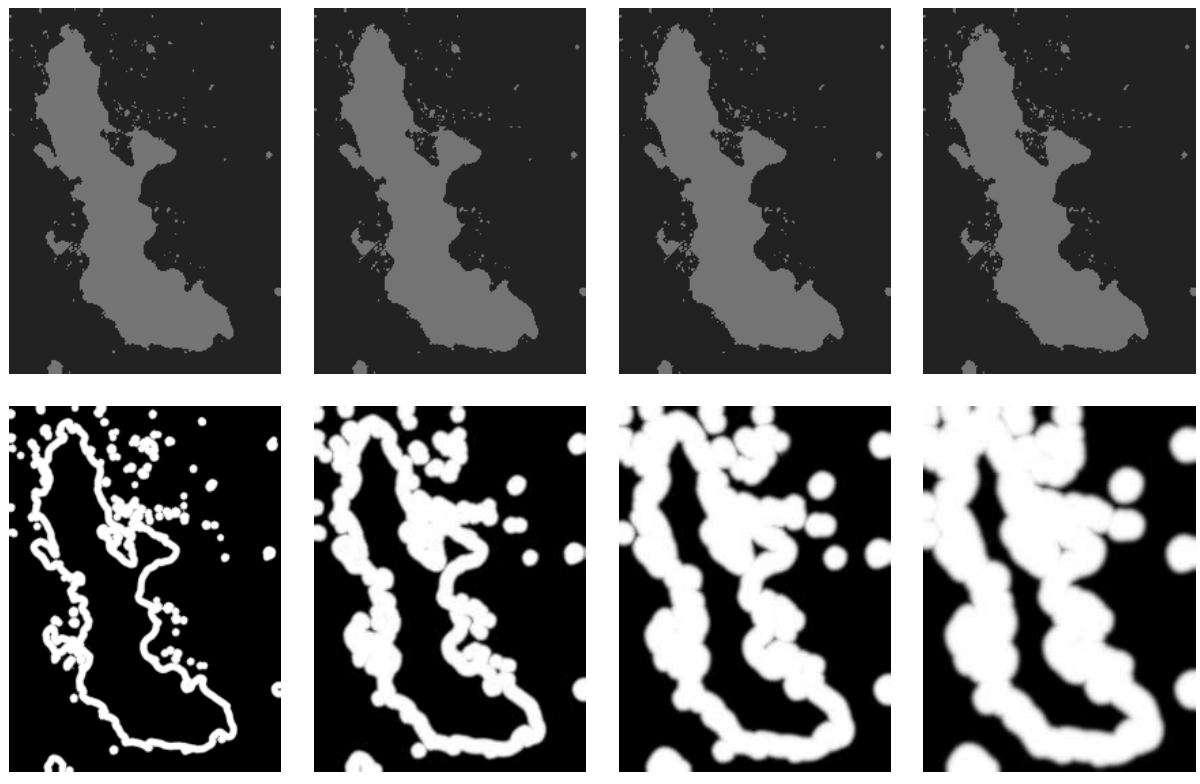

$\varepsilon=20$

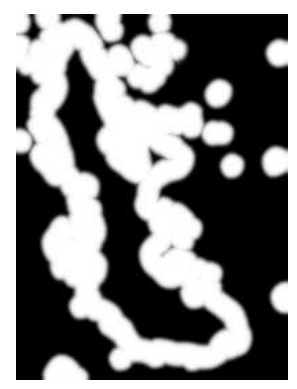

$\varepsilon=30$

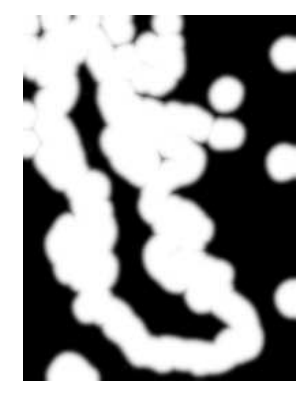

$\varepsilon=40$

FIGURE 4.6. The segmented nebula $u$ and the $\varepsilon$-neighborhood of $\Gamma(u)$, approximated through the Lipschitz level set method, with $\lambda=50$ and $\varepsilon=10,20,30,40$.

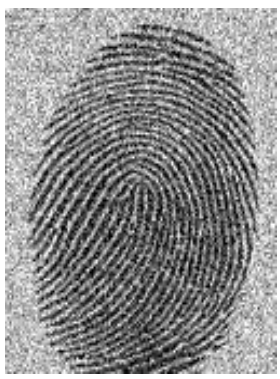

$u_{0}$

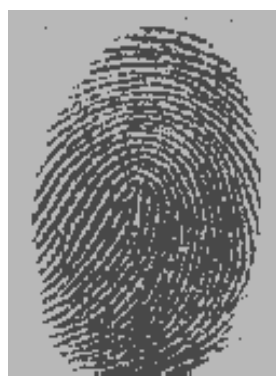

$u$

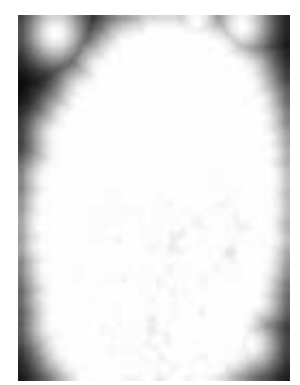

$\varepsilon-\Gamma(u)$

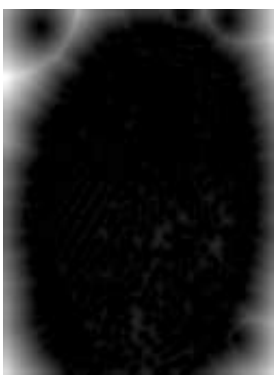

$v$

FiguRE 4.7. The segmented fingerprint using the Ambrosio-Tortorelli approximation with $\lambda=$ 1000 and $\varepsilon=15$. 


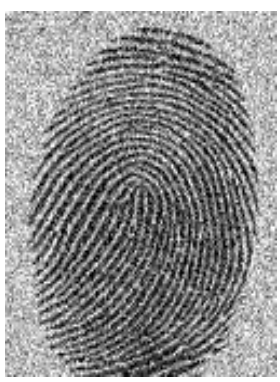

$u_{0}$

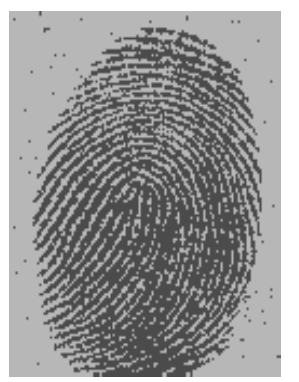

$u$

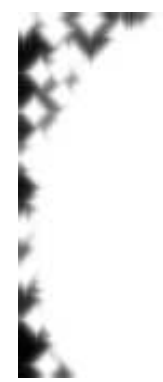

$\varepsilon-\Gamma(u)$

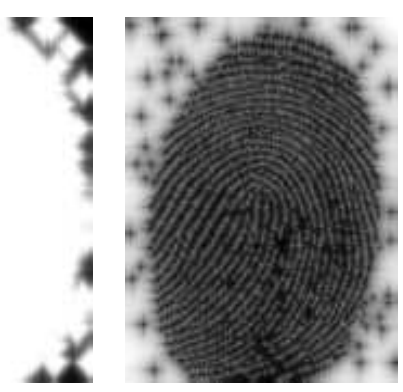

$\phi$

FIGURE 4.8. The segmented fingerprint using the infinite laplacian approximation with $\lambda=20$.

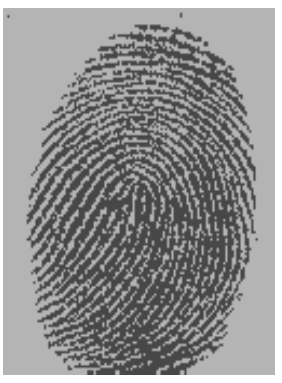

$u_{l s}, \varepsilon=40, \lambda=20$

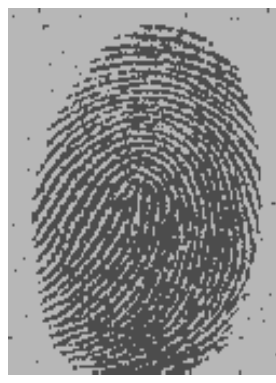

$u_{c v}, \varepsilon=20, \lambda=30$

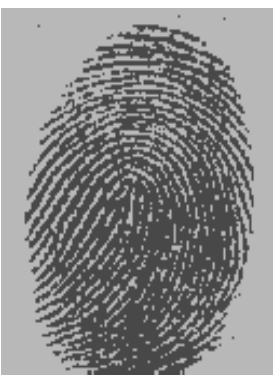

$u_{a t}, \varepsilon=15, \lambda=1000$

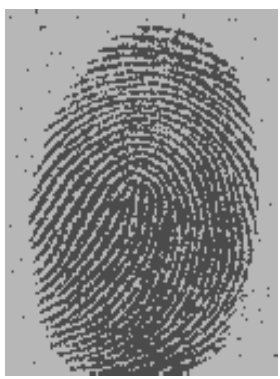

$u_{\infty}, \lambda=20$

FIGURE 4.9. The segmentation results of the fingerprints for the different models.

the same time, tuning $\varepsilon$ we determine the intensity of the denoising effect on dilute noise.

Finally, in Figure 4.7 and Figure 4.8 we show some results concerning the AmbrosioTortorelli approximation and the infinite laplacian approximation. In Figure 4.9 we compare some segmented fingerprint using the Chan-Vese model and our presented model approximated through the Lipschitz level set method, its variant based on the infinite laplacian and the Ambrosio-Tortorelli method.

Acknowledgments. We would like to thank all the visitors of our group "Calculus of Variations and Image processing" and especially Ivano Primi, Antonin Chambolle and the group member Luca Mugnai for constructive discussions and useful remarks.

\section{REFERENCES}

[1] L. Ambrosio and V. M. Tortorelli. Approximation of functionals depending on jumps by elliptic functionals via $\Gamma$-convergence. Comm. Pure Appl. Math., 43(8):999-1036, 1990.

[2] G. Aronsson. Extension of functions satisfying Lipschitz conditions. Arkiv för Matematik, $6(6): 551-561,1967$.

[3] G. Aronsson, M. G. Crandall, and P. Juutinen. A tour of the theory of absolutely minimizing functions. Bull. Amer. Math. Soc. (N.S.), 41(4):439-505, 2004.

[4] E. Bae and X.-C. Tai. Graph cuts for the multiphase mumford-shah model using piecewise constant level set methods. UCLA CAM Report 08-36, 2008.

[5] A. Braides. $\Gamma$-convergence for beginners, volume 22 of Oxford Lecture Series in Mathematics and its Applications. Oxford University Press, Oxford, 2002. 
[6] A. Braides and G. Dal Maso. Non-local approximation of the Mumford-Shah functional. Calc. Var. Partial Differential Equations, 5(4):293-322, 1997.

[7] T. Brox and J. Weickert. Level set based image segmentation with multiple regions. In Pattern Recognition, volume 3175 of Lecture Notes in Computer Science, pages 415-423. Springer Berlin / Heidelberg, 2004.

[8] G. Buttazzo. Semicontinuity, relaxation and integral representation in the calculus of variations, volume 207 of Pitman Research Notes in Mathematics Series. Longman Scientific \& Technical, Harlow, 1989.

[9] V. Caselles, J.-M. Morel, and C. Sbert. An axiomatic approach to image interpolation. IEEE Trans. Image Process., 7(3):376-386, 1998.

[10] T. F. Chan and L. A. Vese. Active contours without edges. IEEE Transactions on Image Processing, 10(2):266-277, 2000.

[11] G. Chung and L. A. Vese. Image segmentation using a multilayer level-set approach. Computing and Visualization in Science, 12(6):267-285, 2009.

[12] C. Elion and L. A. Vese. An image decomposition model using the total variation and the infinity laplacian. volume 6498, page 64980W. SPIE, 2007.

[13] K. J. Falconer. The Geometry of Fractal Sets. Cambridge University Press, Cambridge, 1985.

[14] F. Fraternali, M. Ortiz, and B. Schmidt. Eigenfracture: an eigendeformation approach to variational fracture. Multiscale Model. Simul., 7(3):1237-1266, 2009.

[15] Dal Maso G. An Introduction to Г-Convergence. Birkhäuser, Boston, 1993.

[16] S. Gao and T. D. Bui. Image segmentation and selective smoothing by using Mumford-Shah model. IEEE Transactions on Image Processing, 14(10):1537-1549, 2005.

[17] A. Garroni, V. Nesi, and M. Ponsiglione. Dielecric breakdown: optimal bounds. R. Soc. Lond. Proc. Ser. A Math. Phys. Eng. Sci., 457(2014):2317-2335, 2001.

[18] Y. M. Jung, S. H. Kang, and J. Shen. Multiphase image segmentation via Modica-Mortola phase transition. SIAM J. Appl. Math., 67(5):1213-1232, 2007.

[19] J. Lie, M. Lysaker, and X.-C. Tai. A binary level set model and some applications to MumfordShah image segmentation. IEEE Transactions on Image Processing, 15(5):1171-1181, 2006.

[20] J. Lie, M. Lysaker, and X.-C. Tai. A variant of the level set method and applications to image segmentation. AMS Mathematics of Computation, 75:1155-1174, 2006.

[21] D. Mumford and J. Shah. Optimal approximations by piecewise smooth functions and associated variational problems. Comm. Pure Appl. Math., 42(5):577-685, 1989.

[22] M. Negri. A non-local approximation of free discontinuity problems in $S B V$ and $S B D$. Calc. Var., 25(1):33-62, 2006.

[23] A. M. Oberman. A convergent difference scheme for the infinity Laplacian: construction of absolutely minimizing Lipschitz extensions. Mathematics of Computation, 74(251):12171230, 2005.

[24] S. Osher and J. A. Sethian. Fronts propagating with curvature-dependent speed: algorithms based on Hamilton-Jacobi formulations. J. Comput. Phys., 79(1):12-49, 1988.

[25] B. Sandberg, T. F. Chan, and L. A. Vese. A level-set and gabor-based active contour algorithm for segmenting textured images. UCLA CAM report 02-39, 2002.

[26] J. Shen. Piecewise $H^{-1}+H^{0}+H^{1}$ images and the Mumford-Shah-Sobolev model for segmented image decomposition. AMRX Appl. Math. Res. Express, (4):143-167, 2005.

[27] X.-C. Tai and T. F. Chan. A survey on multiple level set methods with applications for identifying piecewise constant functions. International J. Numer. Anal. Modelling, 1(1):2547, 2004.

[28] L. A. Vese and T. F. Chan. A multiphase level set framework for image segmentation using the mumford and shah model. International Journal of Computer Vision, 50(3):271-293, 2002 . 